\title{
Glucose improves object-location binding in visual-spatial working memory
}

\author{
Brian Stollery $^{1} \cdot$ Leonie Christian ${ }^{1}$
}

Received: 29 July 2015 / Accepted: 19 October 2015 / Published online: 18 November 2015

(C) The Author(s) 2015. This article is published with open access at Springerlink.com

\begin{abstract}
Rationale There is evidence that glucose temporarily enhances cognition and that processes dependent on the hippocampus may be particularly sensitive. As the hippocampus plays a key role in binding processes, we examined the influence of glucose on memory for object-location bindings.

Objective This study aims to study how glucose modifies performance on an object-location memory task, a task that draws heavily on hippocampal function.

Methods Thirty-one participants received 30 g glucose or placebo in a single 1-h session. After seeing between 3 and 10 objects (words or shapes) at different locations in a $9 \times 9$ matrix, participants attempted to immediately reproduce the display on a blank $9 \times 9$ matrix. Blood glucose was measured before drink ingestion, mid-way through the session, and at the end of the session.

Results Glucose significantly improves object-location binding $(d=1.08)$ and location memory $(d=0.83)$, but not object memory $(d=0.51)$. Increasing working memory load impairs object memory and object-location binding, and wordlocation binding is more successful than shape-location binding, but the glucose improvement is robust across all difficulty manipulations. Within the glucose group, higher levels of circulating glucose are correlated with better binding memory and remembering the locations of successfully recalled objects.

Conclusions The glucose improvements identified are consistent with a facilitative impact on hippocampal function. The
\end{abstract}

Brian Stollery

Brian.Stollery@Bristol.ac.uk

1 School of Experimental Psychology, University of Bristol, 12a Priory Road, Bristol BS8 1TU, UK findings are discussed in the context of the relationship between cognitive processes, hippocampal function, and the implications for glucose's mode of action.

Keywords Glucose $\cdot$ Episodic memory $\cdot$ Spatial memory Working memory · Object-location memory · Binding . Complexity $\cdot$ Hippocampus $\cdot$ Human

Glucose is the major energy substrate supporting neuronal functioning (Messier 2004), and it is known that the central nervous system, particularly the hippocampus, is especially vulnerable to interruptions in its supply (Dennis et al. 2011; Tomlinson and Gardiner 2008). Recent comprehensive reviews have concluded that drinking glucose, following an overnight fast, can produce a temporary enhancement of cognition that is often, although not exclusively, seen on episodic memory tasks or cognitive tasks that pose a high level of demand (see Messier 2004; Riby 2004; Smith et al. 2011). While the favoured status of episodic memory can be interpreted in terms of a hippocampal specific action (e.g. McNay and Gold 2001; Riby and Riby 2006; Winocur 1995), only a few human studies have undertaken a focussed evaluation of hippocampal-based cognitive tasks to explore this (e.g. Stollery and Christian 2015).

Given the special status afforded to the hippocampal system for understanding the influence of glucose on cognition, the present study explores glucose's influence on a task widely acknowledged to draw on hippocampal functioning: the objectlocation memory task. In this task, participants maintain in working memory a spatial array of objects (e.g. letters, pictures, or colours) for retrieval. Memory for the objects, the locations of the objects, and the binding of object-location pairs are key performance parameters. The latter memory index is particularly pertinent because an important role of the hippocampus during 
the creation of episodic memories are the binding processes that integrate diverse stimulus attributes (e.g. colour, shape, size, location) into unitary representations (e.g. Mitchell et al. 2000). Indeed, forming and maintaining bound representations is a common activity of the cognitive system (Zimmer et al. 2006) and, as our visual environment contains many objects at different locations, object-location memory is the key feature of working memory enabling us to keep track of the whereabouts of objects in our world (e.g. where is my phone?) and is regarded as the basic binding problem (Treisman 1996).

As the name implies, object-location memory entails remembering what went where and processing visually presented material is organized into two broad functional pathways. The ventral, or "what", pathway is important for visual object recognition and the dorsal, or "where", pathway for the localisation of objects in space (Mishkin and Ungerleider 1982; Ungerleider and Haxby 1994) and one key question concerns the integration of the what and where information streams. Given these two pathway connections to the limbic system and frontal lobes, Mishkin et al. (1983) speculated that one plausible site for this integration is the hippocampal formation. Since that time a range of studies offer broad support for the view that distinct, but interacting (McIntosh and Schenk 2009), neural pathways are involved in remembering object and position information (e.g. Moscovitch et al. 1995; O'Keefe and Nadel 1978) and that hippocampal structures play a critical role in the binding of objects to locations (Bachevalier and Nemanic 2008; Crane and Milner 2005; Finke et al. 2008; Gilbert and Kesner 2004; Hannula and Ranganath 2008; Mumby et al. 2002; Nunn et al. 1999; Olson et al. 2006; Pertzov et al. 2013; Piekema et al. 2006; Postma et al. 2008; Watson et al. 2013). These conclusions are not surprising given the fundamental role of hippocampal structures to relational memory (e.g. Cohen et al. 1999; Olsen et al. 2012, 2013); although, in common with other forms of episodic memory, object-location memory recruits additional neural systems (e.g. prefrontal cortex) to support efficient functioning (see Aggleton 2012; Barker et al. 2007; Barker and Warburton 2015; Lee and Solivan 2008).

Only a few studies have examined the influence of glucose on tasks relevant to the object-location memory. Benton and Owens (1993)showed 16 pictures (e.g. cat, doll) in a $4 \times 4$ grid for $30 \mathrm{~s}$ and after $1 \mathrm{~min}$ of rehearsal-preventing activity, participants relocated the 16 pictures. They found that a $50 \mathrm{-g}$ glucose dose did not influence the speed or accuracy of replacing the pictures. Using the same technique, two related studies report better performance with higher blood glucose levels, with the pictures being located faster and more accurately (Benton and Parker 1998; Benton and Sargent 1992). A later study by Benton and Stevens (2008) examined $25 \mathrm{~g}$ glucose with children aged $9-10$ years. After showing 20 pictures of common objects (e.g. ball, mouse) on a card, the children free-recalled the names of the objects shown. After presenting the same card twice more, with name recall requested after each presentation, the children attempted to relocate the 20 pictures in an empty $5 \times 4$ grid. Those receiving glucose recalled more objects (averaged over the three trials) but did not differ in their placement of the objects. Mohanty and Flint Jr. (2001) presented 16 pictures (all either negative or neutral valence) in a $4 \times 4$ grid for $20 \mathrm{~s}$ and, following $1 \mathrm{~min}$ of rehearsal prevention, participants attempted to place the pictures back in their correct location. This procedure was repeated twice more, using the same picture arrangement, and recall scores averaged. Those given $50 \mathrm{~g}$ glucose took longer to replace the pictures and made more placement errors with the emotional material, but glucose did not influence the performance for the neutral material. In all the above studies, a separate assessment of object memory and location memory is unavailable because all locations contained objects and all the objects were available to the participant during the replacement phase. Thus, the evaluations only consider the correct placement of objects.

Scholey and Kennedy (2004) and Jones et al. (2012) examined location memory by showing participants a house with nine windows, four windows of which were "lit", and tested recognition memory for lit and unlit windows. Unfortunately, by amalgamating different aspects of performance into composite scores (e.g. quality of memory, speed of memory), this study did not evaluate explicitly the effect of glucose on location memory. However, based on the composite scores that incorporate performance on this spatial memory task, glucose either did not influence performance (Scholey and Kennedy 2004) or produce impairment (Jones et al. 2012). Stollery and Christian (2013) also only examined location memory by presenting identical objects (circles) at random locations in a $9 \times 9$ grid and assessing recognition memory for exact locations. Although location memory declines substantially as the number of objects to remember increases, neither speed or accuracy varied with the $50 \mathrm{~g}$ glucose dose given. However, they observed a trend for a slower decline in recognition accuracy with increasing memory load for those given glucose with a drink congruent message.

Finally, three glucose studies have used a computerized version of the Corsi blocks task. Although this task clearly requires location memory, the requirement to reproduce the temporal sequence of locations places additional demands on visual-spatial working memory (Zimmer et al. 2003). As a spatial variant of the verbal digit-span task, typically, evaluations of temporal-spatial memory assess both forward and backward spans (Berch et al. 1998; Brunetti et al. 2014), but glucose studies have only focussed on forward span. SünramLea et al. (2011) found that $25 \mathrm{~g}$ glucose improved performance, but lower $(15 \mathrm{~g})$ and higher $(50$ and $60 \mathrm{~g})$ doses did not. Owen et al. (2012) failed to observe any influence of either a $25-$ or 60 -g glucose dose, following either an overnight fast or a 2-h fast, although a later study found improved 
spans for both a 25 - and 60-g glucose dose following an overnight fast (Owen et al. 2013).

Taken together, there is rather heterogeneous evidence for an impact of glucose on the cognitive processes relevant to objectlocation memory. Moreover, when all locations are used, and participants given the entire set of objects to relocate, the study is unable to evaluate the separate roles of object memory and location memory in successful object-location binding. Additionally, as all locations contain objects, an error in the placement of one object will always lead to an error in the placement of the object originally at that location. More importantly, the sensitivity of the relevant studies to detect glucose related effects is likely to be low for two reasons. First, most studies of object-location memory only use a single presentation-recall trial and the resulting estimates of memory are likely to be less stable compared to those achieved from multiple trials. Second, those studies employing three presentation-recall trials use the same spatial arrangement of objects thereby permitting some learning of the object-location bindings. Here, not only is the extent of learning unassessed but also it is unclear whether this learning would be expected to weaken or strengthen possible glucose effects.

Given the currently important role that the hippocampus plays in understanding glucose-related enhancements of episodic memory, the present study evaluates the influence of glucose on the hippocampal-dependent object-location memory task. To enable a reasonably broad initial evaluation, we incorporated several changes to the basic paradigm. First, multiple trials, each trial using a new spatial arrangement of objects, are given to provide a more stable estimate of memory. Second, manipulating the number of objects to remember allows an examination of working memory load and this addresses issues pertinent to the influence of task complexity on glucose-related changes. Third, using a larger $(9 \times 9)$ grid, coupled with a maximum of ten object-locations to remember, ensures that on each trial not all potential locations contain an object. Fourth, two versions of the object-location task are deployed for evaluating the generality of any effects found. In one version, the objects are common words, with their rich semantic associations. In the other version, the objects are relatively simple geometrical shapes. Finally, the free-recall data collected enables separate evaluations of memory for the objects, memory for the locations, and their joint contribution to successful object-location binding.

\section{Methods}

\section{Participants}

Thirty-two participants completed a single-session study lasting about $60 \mathrm{~min}$. No participants had diabetes or phenylketonuria, and all were fluent in English and had normal or corrected vision. Participants were required to fast from midnight the previous night and only drink water prior to attending the morning session to ensure their blood glucose was at fasting levels. The University Research Ethics Committee approved the study, and all participants gave written informed consent prior to their participation in the study. At the end of the study, participants were asked to confirm their consent for the data to be used. Based on our study design, power calculations showed that a total of 32 participants were needed to detect $(p=0.05)$ a medium glucose effect $\left(d=0.50, \eta_{\mathrm{p}}{ }^{2}=0.06\right)$ with $95 \%$ power (Faul 2007). However, data from one participant (placebo group) was excluded because they failed to confirm consent for their data to be used, leaving a sample size of 31 participants (see Table 1).

\section{Procedure}

Participants arrived for testing at about 09:30, re-read the recruitment information, had any questions answered, provided informed consent, and the exp26rimenter administered the drink (glucose or placebo) according to a predetermined random order. A maximum of three participants were tested on each session. The drinks comprised $300 \mathrm{ml}$ of water mixed with $30 \mathrm{ml}$ of "no added sugar" orange and lemon squash. The glucose drink contained $30 \mathrm{~g}$ glucose $(114 \mathrm{kcal}$ or $477 \mathrm{~kJ})$, and the placebo drink contained $45 \mathrm{mg}$ saccharin $(1.8 \mathrm{kcal}$ or $7.8 \mathrm{~kJ})$. Earlier work has shown that this results in a similar "mouth feel" and sweetness for the two drinks (e.g. Meikle et al. 2004). Following consent, participants had their blood glucose measured (presession) using the OneTouch Ultra blood glucose monitoring system (donated by Johnson \& Johnson Company) and then received their allocated drink (glucose or placebo). While they waited $10 \mathrm{~min}$ to allow blood glucose levels to rise (Meikle et al. 2004; Stollery and Christian 2013), demographic information was collected (e.g. age, sex, BMI, fasting compliance) and they completed a stress-arousal checklist (Mackay et al. 1978) to evaluate whether stress or arousal changes mediate the effect of glucose on performance (see Meikle et al. 2004; Smith et al. 2011). They then began their first version of the object-location binding task (either the shape or word version). After finishing that version (about $20 \mathrm{~min}$ ), there was a brief rest break during which a second (mid-session) blood glucose measure was taken. The participants then began the other version of the objectlocation binding task. After finishing the task, participants completed the stress-arousal checklist, made a forced choice decision about the drink they believed they had consumed, and the final blood glucose measure taken. Finally, they were thanked, received debriefing information, and reconfirmed their consent.

\section{The object-location binding task}

Two versions of a computerized object-location binding task were used: one version used words as objects and the other used shapes (see Fig. 1). In both versions, each 
Table 1 Basic demographic information, blood glucose values, within-session timings, and overall performance on the object-location binding task $( \pm \mathrm{SE})$

\begin{tabular}{lcc}
\hline & Glucose $(n=16)$ & Placebo $(n=15)$ \\
\hline Female/Male ratio & $11 / 5$ & $11 / 4$ \\
Age (years) & $22.5(1.5)$ & $26.5(4.0)$ \\
Body mass index & $21.6(0.6)$ & $23.2(0.9)$ \\
Self-reported mood & & \\
Pre-session stress & $4.9(1.1)$ & $2.9(1.1)$ \\
Post-session stress & $4.0(0.8)$ & $4.6(0.8)$ \\
Pre-session arousal & $5.1(0.9)$ & $5.9(0.9)$ \\
Post-session arousal & $6.8(1.0)$ & $7.1(1.0)$ \\
Blood glucose (mmol/L) & & \\
Pre-session & $5.16(0.14)$ & $5.99(0.14)$ \\
Mid-session & $7.84(0.23)$ & $5.15(0.24)$ \\
Post-session & $7.81(0.30)$ & $5.11(0.31)$ \\
Within-session timings (min) & & $33.13(1.47)$ \\
Pre- to mid-session & $31.31(1.43)$ & $20.80(0.95)$ \\
Mid- to post-session & $20.81(0.92)$ & $53.93(2.07)$ \\
Average session length & $52.13(2.01)$ & \\
Overall task performance & & $63.06(2.68)$ \\
Object memory (\%) & $67.38(1.54)$ & $25.76(1.54)$ \\
Location memory (\%) & $30.35(1.29)$ & $16.25(1.00)$ \\
Object-location binding memory (\%) & $20.83(1.15)$ & $29.31(2.11)$ \\
Retrieval time (s) & $27.30(1.72)$ & $0.68(0.06)$ \\
Number of invalid location errors & $1.96(0.13)$ & $0.43(0.02)$ \\
Number of location swap errors & $0.70(0.05)$ & \\
Mean conditional probability & $0.47(0.02)$ & $*$ \\
\hline & & \\
$* *$ \\
$*$
\end{tabular}

Glucose effects: $\# p=0.056 ;{ }^{*} p<0.05 ; * * p<0.01$ presentation-recall trial followed the same procedure. For the presentation phase, an empty $9 \times 9$ matrix was displayed continuously on a $43-\mathrm{cm}$ monitor. The four corners of the $9 \times 9$ matrix were not used to display objects, leaving 77 possible display locations. Each trial began with the simultaneous presentation of several objects (memory load) at randomly selected matrix locations (each $25 \times 15 \mathrm{~mm}$ ) with instructions to remember the objects and their location. Objects were centred in their matrix location, and participants were free to position themselves in front of the monitor at a comfortable viewing distance. Four memory loads were used (i.e. 3, 5,7 , and 10 objects), and the total presentation time was adjusted for the number of objects displayed: $2 \mathrm{~s}$ plus $1.5 \mathrm{~s}$ per object (i.e. $6.5,9.5,12.5$, and $17.0 \mathrm{~s}$ respectively across the four memory loads).

Immediately after the objects disappeared the recall phase began. For each version, participants were provided with a 24-page booklet, placed at the side of the monitor, and each page contained an empty $9 \times 9$ matrix. Using this empty matrix, participants were asked to write down all the objects they saw in their remembered locations, as quickly and accurately as possible. If the participant could recall an object but they were uncertain about the object's exact location, they were asked to write the object at the location that represented "their best guess". If a participant could recall the location of an object, but they could not retrieve any information about the object's identity, then they simply wrote an $\mathrm{X}$ at the remembered location in the blank $9 \times 9$ matrix. After completing this timed free-recall phase, participants turned over to the next page of the booklet and initiated the next trial by pressing the space bar on a standard keyboard. The next presentation-recall trial began after 2 s. No feedback was provided.

The order of the word and shape versions of the task was counterbalanced across participants and each version lasted about $20 \mathrm{~min}$. The task performance was assessed using five main indicators: correctly recalled objects, correctly recalled locations, correctly recalled objects recalled in the correct location, the time taken to recall this information, and placement errors. All performance measures were averaged across the six replications employed (see next paragraph). 
(a) word location binding task

\begin{tabular}{|l|l|l|l|l|l|l|l|l|}
\hline & Copper & & & & & & & \\
\hline & & & & Pastor & & & & \\
\hline & & & & & & & & \\
\hline & & & & & & & Budget & \\
\hline & & & & & & & & \\
\hline & Nature & & & & & & & \\
\hline & & & & & & Lotion & & \\
\hline & & & Secure & & & & & \\
\hline & Planet & & & & & & & \\
\hline
\end{tabular}

(b) shape location binding task

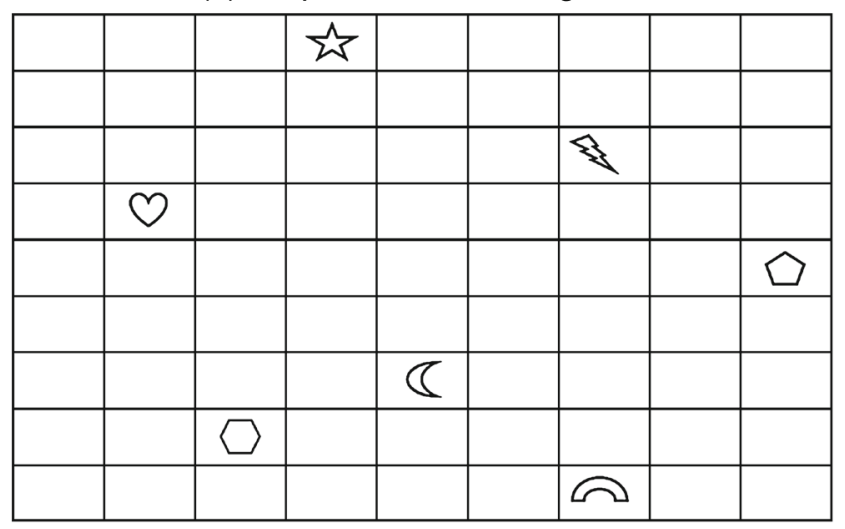

Fig. 1 Object-location binding task with a memory load of seven objects for $\mathbf{a}$ word and $\mathbf{b}$ shape versions

Each version of the object-location binding task comprised 24 presentation-recall trials arranged as six blocks, each block containing four trials. The four trials within a block comprised one trial at each of the four memory loads (i.e. 3, 5, 7, and 10 objects) in random order. The six blocks were replications of the memory load factor and blocks followed each other without a break. For the word version, the stimuli comprised a pool of 150 words created using the MRC psycholinguistic database (Fearnley 1997). All words were six letters long, contained between 1 and 3 syllables (2.0 \pm 0.34$)$, and had mid-range values for imagability (444 \pm 8.0 ), concreteness $(433 \pm 9.9)$, meaningfulness $(397 \pm 8.0)$, and Thorndike-Lorge written frequency $(130 \pm 13.5)$; all values shown are mean $\pm \mathrm{SE}$. Words were displayed in Courier 14 font and only used once. Each participant saw a different random selection from the word pool across the 24 presentation-recall trials. For the shape version, the stimuli comprised 25 geometric forms (e.g. triangle, circle, heart, and hexagon). On each trial, shapes were randomly selected such that each shape was only used once within the set of four trials that defined a replication block. The 25 shapes were reused on each of the six blocks.

\section{Statistical analysis}

The main design for the cognitive analysis is a threefactor mixed ANOVA, with drink (glucose or placebo) as the unrelated factor and object type (word vs. shape) and memory load $(3,5,7$, and 10) as the two related factors. The partial eta-square $\left(\eta_{\mathrm{p}}{ }^{2}\right)$ effect size for glucose is cited $(0.01 \cong$ small, $0.06 \cong$ medium, $0.15 \cong$ large $)$ with the $F$-ratio and the Cohen's $d$ effect size is also provided for selected comparisons. When sphericity violations occur, the Huynh-Feldt corrected $p$ values are reported, but the original degrees of freedom are cited for readability. The Tukey (HSD) test is used for post hoc pair-wise comparisons.

\section{Results}

The results from the study are presented in four main sections. The first considers evidence relating to drink detection, group matching, blood glucose changes, and the assessment of stress and arousal. In the second, basic performance measures from the object-location task are reported (e.g. item memory). The third presents a conditional probability analysis to examine the relative importance of object and location memory to successful object-location binding. Finally, the influence of blood glucose measures on object-location performance is considered.

\section{Drink detection, blood glucose, and mood}

Based on the forced-choice decision participants made at the end of their session, participants were unable to identify the drink they received $\left(\chi^{2}(1, N=31)=0.32, p=\right.$ $0.570)$; although the majority ( $84 \%$ ) believed they had consumed glucose. As shown in Table 1, the glucose and placebo groups did not differ in age $(t(29)=0.96$, $p=0.345)$, body mass index $(t(29)=1.53, p=0.137)$, or in the distribution of male/female participants $\left(\chi^{2}(1)=\right.$ $0.079, p=0.779)$. A series of Pearson correlations between the demographic, mood, and object-location memory performance showed no significant correlations, indicating that the performance was not related to variations in age, BMI, or mood.

\section{Blood glucose changes and timing}

Blood glucose levels were analysed using a two-factor mixed ANOVA with drink (glucose, placebo) as the unrelated factor and time (pre-session, mid-session, post-session) as the related factor. There were main effects of drink $(F(1,29)=45.6$, $p<0.001)$, time $(F(1,29)=47.0, p<0.001)$ and a drink $\times$ time 
interaction $(F(2,58)=38.3, p<0.001 ; \mathrm{MSE}=0.434)$. Post hoc analysis showed no pre-session differences between the glucose and placebo groups, no change in blood glucose across time for the placebo group but raised levels for the glucose group at mid-session $(p<0.01)$ that remained constant until the end of the session (see Table 1).

As the object-location task was self-paced, the relative timing of the mid- and post-session glucose measurements was evaluated (see Table 1). This showed the mid-session measurement occurred about $32 \mathrm{~min}$ after the pre-session measurement, with the post-session measurement about 21 min later, giving an average session duration of $53 \mathrm{~min}(\mathrm{SD}=8 \mathrm{~min})$. The longer average interval from pre- to mid-session was due to the 10-min waiting period following glucose ingestion prior to commencing the object-location task. Analysis of the timings showed no difference in average session duration as a function of drink ( $F(1$, 29) $=0.393, p=0.535$ ), and there were no differences in the relative timing of the mid- and post-session timings for the glucose and placebo groups $(F(1,29)=0.937, p=0.341)$.

\section{Self-reported mood}

Subjective evaluations of stress and arousal were analysed using a two-factor mixed ANOVA with drink (glucose, placebo) as the unrelated factor and time (pre-session, post-session) as the related factor (see Table 1). There was no evidence that glucose consumption influences stress or arousal. For arousal, there was no main effects of drink $(F(1,29)=0.286, p=0.587)$, a tendency for higher arousal at the end of the session (5.5 vs. 6.9; $F(1,29)=3.43, p=0.070)$, and no interaction $(F(1,29)=$ $0.132, p=0.719)$. For stress, there were no effects of drink $(F(1,29)=0.451, p=0.507)$, time $(F(1,29)=0.271, p=0.606)$, and no time $\times$ drink interaction $(F(1,29)=3.06, p=0.091)$.

\section{Object-location memory task}

As shown in Table 2, task performance was assessed using five primary indicators: the percentage of objects correctly recalled (object memory), the percentage of locations correctly recalled (location memory), the percentage of objects correctly recalled in the correct location (object-location binding memory), the time taken to place the recalled objects in their remembered locations (retrieval time), and placement errors. Table 1 also summarizes overall object-location performance, for each drink condition, on the different performance measures analysed.

Initially, a check was made for changes in object memory across the six blocks of trials. This was relevant because although each block contained unique words, the 25 shapes were reused in each block. There was no evidence that object memory varied across the six blocks $(F(5,150)=0.276, p=$ $0.881)$; there was no overall advantage for recalling words or shapes $(F(1,30)=2.80, p=0.105)$ and no object type $\times$ block
Table 2 Descriptive statistics for the primary outcome measures in the object-location memory task ( \pm SE)

\begin{tabular}{|c|c|c|c|c|}
\hline & \multicolumn{2}{|l|}{ Glucose } & \multicolumn{2}{|l|}{ Placebo } \\
\hline & Words & Shapes & Words & Shapes \\
\hline \multicolumn{5}{|c|}{ Object memory (\%) } \\
\hline 3 objects & $96.9(2.5)$ & $85.8(2.8)$ & $91.9(2.6)$ & $83.3(2.9)$ \\
\hline 5 objects & $79.4(3.6)$ & $69.8(3.7)$ & $71.1(3.7)$ & $68.0(3.8)$ \\
\hline 7 objects & $58.2(3.5)$ & $58.0(3.6)$ & $56.0(3.6)$ & $52.2(3.8)$ \\
\hline 10 objects & $46.5(2.6)$ & $44.6(2.6)$ & $40.9(2.7)$ & $41.2(2.7)$ \\
\hline \multicolumn{5}{|c|}{ Location memory (\%) } \\
\hline 3 objects & $50.0(3.9)$ & $26.0(2.7)$ & $40.4(4.1)$ & $23.4(2.8)$ \\
\hline 5 objects & $34.6(3.3)$ & $24.8(2.1)$ & $28.0(3.4)$ & $22.9(2.2)$ \\
\hline 7 objects & $30.2(2.9)$ & $22.9(2.2)$ & $28.8(3.0)$ & $19.5(2.3)$ \\
\hline 10 objects & $32.8(2.6)$ & $22.5(1.6)$ & $25.2(2.7)$ & $19.9(1.6)$ \\
\hline \multicolumn{5}{|c|}{ Object-location binding memory (\%) } \\
\hline 3 objects & $46.2(3.7)$ & $21.2(2.4)$ & $37.0(3.9)$ & $19.6(2.5)$ \\
\hline 5 objects & $27.1(2.8)$ & $15.4(2.2)$ & $16.7(2.9)$ & $14.4(2.3)$ \\
\hline 7 objects & $18.3(2.4)$ & $12.4(1.7)$ & $15.7(2.5)$ & $08.7(1.8)$ \\
\hline 10 objects & $17.8(2.2)$ & $08.3(1.1)$ & $10.8(2.2)$ & $07.1(1.2)$ \\
\hline \multicolumn{5}{|c|}{ Retrieval time (s) } \\
\hline 3 objects & $16.7(1.2)$ & $18.0(1.4)$ & $16.9(1.3)$ & $17.9(1.4)$ \\
\hline 5 objects & $26.1(2.5)$ & $25.9(2.1)$ & $28.1(2.5)$ & $27.2(2.2)$ \\
\hline 7 objects & $29.9(2.4)$ & $31.9(2.8)$ & $33.2(2.5)$ & $33.4(2.9)$ \\
\hline 10 objects & $34.5(2.6)$ & $35.5(2.9)$ & $38.2(2.6)$ & $39.5(3.0)$ \\
\hline
\end{tabular}

interaction $(F(5,150)=0.862, p=0.476)$. Thus, there was no evidence for either learning or a build-up of proactive inhibition across the six blocks and the accuracy of recalling the word and shape stimuli was comparable.

\section{Object memory}

The main effect of drink was not significant $(F(1,29)=2.02$, $p=0.166 ; \eta_{\mathrm{p}}{ }^{2}=0.065$ ) but did show a medium-size advantage for glucose ( 67 vs. $63 \% ; d=0.511)$. Moreover, drink did not interact with object type $(F(1,29)=0.192, p=0.664)$, memory load $(F(3,87)=0.065, p=0.978)$, or the object type $\times$ memory load interaction $(F(3,87)=1.02, p=0.390)$. Object memory declined progressively with increasing memory load $(F(3$, $67)=335.2, p<0.001, d=6.11)$, and the main effect of object type $(F(1,29)=4.92, p=0.035)$ interacted with memory load $(F(3,87)=3.98, p=0.010 ; \mathrm{MSE}=67.37)$.

Post hoc analysis localized the source of the interaction to the two smallest memory loads, where words were better recalled than shapes (three objects, $p<0.01$; five objects, $p=$ 0.05 ). The recall of words and shapes did not differ for seven and ten objects. Both words and shapes showed a progressive decline in accuracy with increases in memory load (all $p<0.01)$. 


\section{Location memory}

Those consuming glucose were more accurate in recalling the location of objects ( 30 vs. $26 \% ; F(1,29)=5.28, p=0.029$; $\left.\eta_{\mathrm{p}}{ }^{2}=0.154 ; d=0.826\right)$, but drink did not interact with object type $(F(1,29)=1.01, p=0.323)$, memory load $(F(3,87)=0.162$, $p=0.907)$, or the object type $\times$ memory load interaction $(F(3$, $87)=0.384, p=0.752$ ) indicating a general benefit of glucose in accurately recalling locations. Location memory was better for words than shapes (33 vs. $23 \% ; F(1,29)=29.8, p<0.001)$, and the main effect of memory load $(F(3,87)=14.1, p<0.001)$ interacted with object type $(F(3,87)=8.32, p<0.001$; $\mathrm{MSE}=$ 71.95).

Post hoc analysis of the interaction localized the source to the three-word memory load. Memory for the location of words was better for three compared to five words (45 vs. $31 \%, p<0.01$ ) and thereafter remained constant at $29 \%$. For shapes, location memory did not vary with memory load $(25,24,21$, and $21 \%$, respectively). Thus, location memory was constant across memory loads, with the exception of the three-word condition where there was better location memory. The better location memory for words was present at all memory loads (three objects, $p<0.01$, and all other memory loads, $p<0.05)$.

\section{Object-location binding memory}

Those receiving glucose showed better object-location binding $\left(21\right.$ vs. $16 \% ; F(1,29)=8.94, p=0.006 ; \eta_{\mathrm{p}}{ }^{2}=0.326 ; d=$ $1.075)$, and this benefit did not interact with object type $(F(1,29)=2.32, p=0.139)$, memory load $(F(3,87)=0.30, p=$ $0.787)$, or the object type $\times$ memory load interaction $(F(3$, $87)=1.22, p=0.305$ ), indicating a general advantage of glucose on binding effectiveness. In addition, word-location binding was more successful than shape-location binding $(F(1,29)=33.2, p<0.001)$; binding became generally less efficient with increasing memory load $(F(3,87)=68.7, p<0.001)$; and there was an object type $\times$ memory load interaction $(F(3$, $87)=12.2, p<0.001 ; \mathrm{MSE}=78.17$ ).

Post hoc analysis of the interaction localized the source to the smallest memory load. When three objects were shown, word-location binding was better than shape-location binding (42 vs. $20 \%, p<0.01$ ). When five objects were presented, word-location binding declined by $20 \%(p<0.01)$; shapelocation binding showed a marginal decline $(5.5 \%$, critical difference $=7.02 \%$ for $p<0.05$ ), with word-location binding remaining superior. As memory load increased from five to ten objects, word-location binding remained superior to shape-location binding $(F(1,29)=16.0, p<0.001, d=1.03)$ and binding effectiveness declined $(F(2,58)=19.3, p<0.001$, $d=1.21)$ at the same rate for words and shapes $(F(2,58)=0.02$, $p=0.979$ ).

\section{Retrieval time}

Retrieval times did not differ as a function of drink $(F(1,29)=$ $0.55, p=0.463)$ and object type $(F(1,29)=0.30, p=0.590)$ but increased with memory load $(F(3,87)=144.7, p<0.001)$. No other effects approached significance and, in particular, drink did not interact with object type $(F(1,29)=0.05, p=0.820)$, memory load $(F(3,87)=1.29, p=0.283)$, or the object type $\times$ memory load $(F(3,87)=0.31, p=0.816)$ interaction. Post hoc analysis simply showed that the time taken to recall objects increased with the number of objects to recall $(17,27,32$, and 37 s respectively; all $p<0.01$ ).

In summary, as shown in Table 1 , those receiving glucose showed a particularly strong facilitative effect on objectlocation binding $(d=1.075)$ and location memory $(d=0.826)$, with only weak evidence for improved object memory $(d=$ 0.511). Increasing the number of objects to remember had a pronounced and detrimental impact on object memory and a smaller, but reliable, decrement in object-location binding. Except for the three-word condition, location memory did not vary with memory load. Although location memory for words was better than for shapes and word-location binding was generally more successful than shape-location binding, none of these effects varied with the consumption of glucose. Thus, the benefits of glucose on object-location binding and location memory did not vary according to task difficulty as assessed by changes in memory load or the type of object to remember. The invariance of the glucose effect across the different memory loads for object memory, location memory, and object-location binding is illustrated in Fig. 2.

\section{Object-location errors}

Given the superior recall of objects compared to locations, there are two important kinds of error to consider for the misplacement of correctly recalled objects. Participants can place a recalled object at either an unused location (invalid location error) or a valid location that is invalid for that object (location swap error). While both errors represent failures in selecting the correct location of a valid object, the invalid location error has no subsequent impact on task performance because the selected location did not contain an object. In contrast, location swap errors entail placing an object at the location of another object. It follows that subsequently recalling the object originally at that location must also give rise to an error because a different object now occupies the valid location. To explore the influence of glucose on these two kinds of error, a four-factor mixed ANOVA with error type (invalid location, location swap), object type (word, shape), and memory load $(3,5,7$, and 10) as the related factors and drink (glucose, placebo) as the unrelated factor was conducted on the number of errors made. 


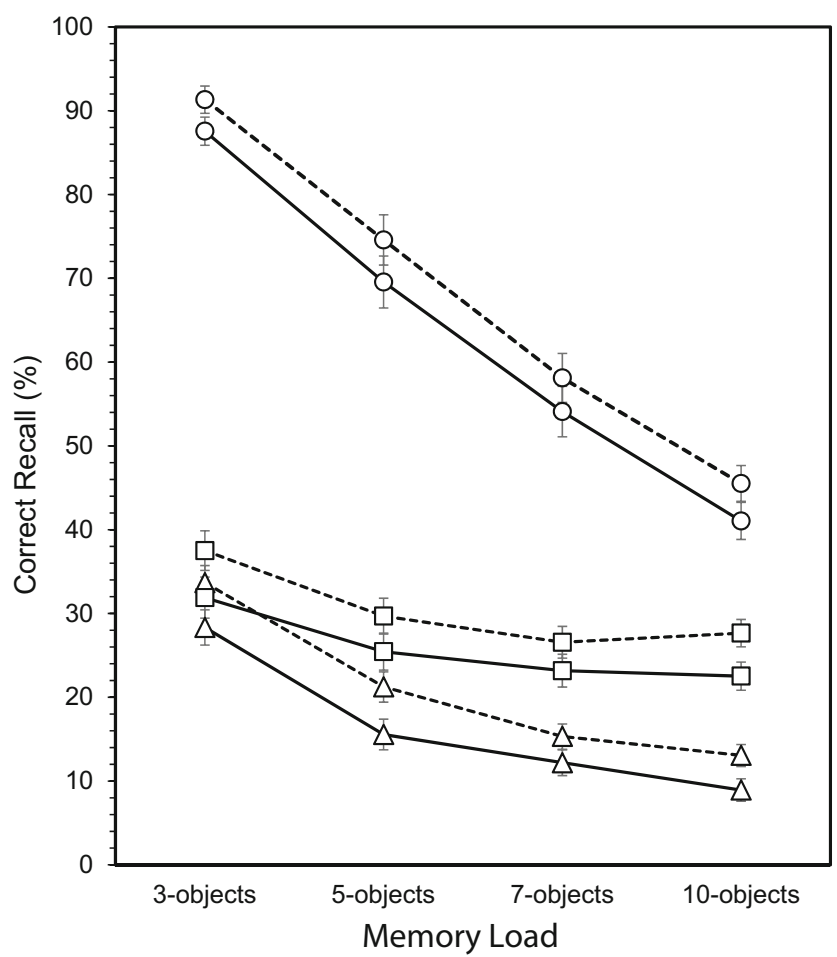

Fig. 2 Object, location, and binding memory as a function of memory load and glucose consumption $( \pm \mathrm{SE})$. Dashed line glucose, solid line placebo. $\circ$ item memory, $\square$ location memory, $\Delta$ object-location binding memory

The analysis shows no main effect of drink $(F(1,29)=.001$, $p=0.986)$ and none of the interactions with drink approach significance. The most common error was placing the recalled object at an invalid location (1.97 vs. $0.69, F(1,29)=72.0$, $p<0.001)$, and more errors were made when the object was a shape $(1.41$ vs. $1.25, F(1,29)=5.10, p=0.032)$. The general increase in errors with memory load $(F(3,87)=66.4, p<0.001)$ further interacted with (a) error type $(F(3,87)=23.6, p<0.001$; $\mathrm{MSE}=0.470)$ and $(\mathrm{b})$ object type $(F(3,87)=2.94, p=0.037$; $\mathrm{MSE}=0.133)$. Neither the error type $\times$ object type $\times$ memory load $(F(3,87)=1.83, p=0.153)$ or its interaction with drink $(F(3,87)=0.38, p=0.738)$ was significant.

As shown in Fig. 3, post hoc analysis of the error type $\times$ memory load interaction showed invalid location errors exhibited a shallow inverted U-shaped effect across memory load: errors increased from 3 to 5 objects $(p<0.01)$, showed no change from 5 to 7 objects, and then showed a small decline from 7 to 10 objects $(p=0.050)$. In contrast, the number of location swap errors increased progressively across memory load $(p<0.01)$. Invalid location errors were more common than location swap errors at each memory load.

For the object type $\times$ memory load interaction, post hoc analysis localized the source to the highest memory load. Words and shapes produced equivalent binding errors for 3 , 5 , and 7 objects, but shapes showed more binding errors for 10 objects $(p<0.01)$.

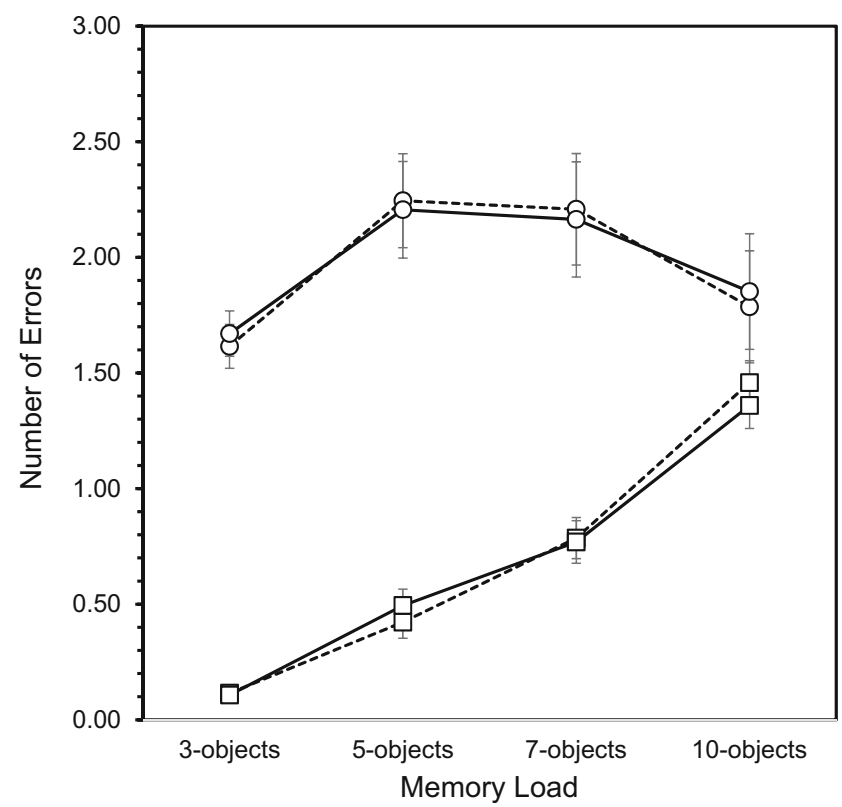

Fig. 3 Object-location binding errors $( \pm$ SE) for the glucose and placebo groups. Dashed line glucose, solid line placebo. $~ \circ$ invalid location error, $\square$ location swap error

In conclusion, the most common binding error was placing recalled objects at an invalid location, words showed the same kinds of binding errors as shapes (except at the highest memory load), and the two kinds of binding error showed distinct influences of memory load. However, there were no effects of glucose on the incidence or type of error made.

\section{Conditional probability analysis}

The above analyses indicated that glucose preferentially improves location memory and object-location binding memory but did not influence the nature and type of binding errors made. Next, we examine the relative importance of object identity and object location in this successful binding using a conditional probability analysis. This analysis examines the probability of one event occurring, given that another event has already occurred. In the context of the current task, when binding two attributes of an object together, the probability of recalling one attribute (e.g. the object) can be conditional on the ability to recall the other attribute (e.g. the location). Thus, there are two conditional probabilities of interest. The first is the probability of recalling the object, given that the location has been successfully recalled-given by $p(\mathrm{O} \mid \mathrm{L})$. The second is the probability of recalling the location, given that the object has been successfully recalled-given by $p(\mathrm{~L} \mid \mathrm{O})$. As these two conditional probabilities need not be symmetric, especially in light to the special status of location memory (e.g. Caprio et al. 2010), the following analyses examine the impact of glucose on both conditional probabilities.

A four-factor mixed ANOVA with drink (glucose, placebo) as the unrelated factor and conditional probability type 
$(p(\mathrm{O} \mid \mathrm{L}), p(\mathrm{~L} \mid \mathrm{O}))$, object type (word, shape), and memory load $(3,5,7$, and 10$)$ as the three related factors was conducted on the conditional probabilities (see Table 3). Average conditional probabilities tended to be higher for those receiving glucose (0.471 vs. $0.425 ; F(1,29)=3.97, p=0.056 ; \eta_{\mathrm{p}}{ }^{2}=0.120 ; d=$ $0.730)$. Although the glucose effect size was numerically greater for $p(\mathrm{~L} \mid \mathrm{O})$ than $p(\mathrm{O} \mid \mathrm{L})(d=0.654$ vs. 0.508$)$, there was no evidence that drink interacted with conditional probability type $(F(1,29)=0.003, p=0.960)$, object type $(F(1,29)=$ $2.59, p=0.118)$, and memory load $(F(3,87)=0.885, p=0.452)$; and none of the glucose interactions approached significance.

The main effect of conditional probability type $(F(1,29)=$ $\left.381, p<0.001 ; \eta_{\mathrm{p}}{ }^{2}=0.929 ; d=4.439\right)$ showed that the probabilities were asymmetric: correctly recalling a location more effectively predicted the probability of recalling the object at that location $(p(\mathrm{O} \mid \mathrm{L})=0.621)$ than correctly recalling an object predicted the probability of recalling the location of that object $(p(\mathrm{~L} \mid \mathrm{O})=0.275)$. Conditional probabilities were also generally higher for words than shapes ( 0.506 vs. $0.390 ; F(1,29)=35.5$, $p<0.001, d=1.344)$, and the main effect of memory load ( $F(3$, $87)=58.7, p<0.001, d=3.518)$ entered into two interactions: a conditional probability type $\times$ memory load interaction $(F(3$, $87)=76.8, p<0.001$ ) which further interacted with object type $(F(3,87)=3.40, p=0.021)$.

Follow-up analysis of the three-way interaction showed the basic memory load $\times$ object type interaction was restricted to $p(\mathrm{~L} \mid \mathrm{O})$ : the probability of recalling the correct location, conditional on successful object recall. Specifically, as shown in Fig. 4, $p(\mathrm{~L} \mid \mathrm{O})$ was higher for words than shapes $(F(1,29)=$ 27.6, $p<0.001)$, and the main effect of memory load $(F(3$, $87)=9.79, p<0.001)$ interacted with object type $(F(3,87)=$ $3.72, p=0.014 ; \mathrm{MSE}=0.012)$. In contrast, although $p(\mathrm{O} \mid \mathrm{L})$ was also higher for words $(F(1,29)=18.0, p<0.001)$ and declined with memory load $(F(3,87)=87.3, p<0.001)$, the object type $\times$ memory load interaction did not approach significance $(F(3,87)=0.155, p=0.926)$.

Post hoc analysis showed that the object type $\times$ memory load interaction for $p(\mathrm{~L} \mid \mathrm{O})$ was due to the three-word condition. Given successful word recall, the probability of recalling the location of that word declined from three words to five words $(p<0.01)$ and then remained constant. For shapes, the probability of recalling the location of a correctly recalled shape did not vary with memory load. Furthermore, for memory loads of five and more objects, $p(\mathrm{~L} \mid \mathrm{O})$ was higher for words $(F(1,29)=17.6, p<0.001)$, showed no effect of memory load $(F(2,58)=0.061, p=0.941)$, and no object type $\times$ memory load interaction $(F(2,58)=1.73, p=0.186)$.

\section{Correction for guessing}

The possibility that $p(\mathrm{~L} \mid \mathrm{O})$ was overestimated due to guessing needs to be considered. On some trials, an object (e.g. a star) can be correctly recalled, but there is uncertainty about the object's exact location. In these situations, the participant's guess can place the recalled object at the correct location, the location of another object (location swap error), or at an unused location (invalid location error). In the current study, invalid location errors were the dominant error, although location swap errors are an established binding error (see Pertzov et al. 2013; Postma and De Haan 1996; Watson et al. 2013). The problem with estimating $p(\mathrm{~L} \mid \mathrm{O})$ arises when the guess places the object at one of the valid locations because then the observed proportion of location correct trials will overestimate the "true" value of $p(\mathrm{~L} \mid \mathrm{O})$. One solution to this, proposed by Dent and Smyth (2005), assumes that when uncertain of a recalled object's location, a random choice is made from all the available locations. Explicitly, with 77 possible object locations, participants can correctly guess the location of a correctly recalled object with a probability of 0.0129 (i.e. 1 in 77). According to Dent and Smyth (2005), p. 122 the true $p(\mathrm{~L} \mid \mathrm{O})$ is therefore given by the following equation: $1+((n(p(\mathrm{~L} \mid \mathrm{O})-1)) /(\mathrm{n}-1))$, where $n$ is the total number of locations to guess from (i.e. 77) and $p(\mathrm{~L} \mid \mathrm{O})$ is the uncorrected conditional probability. With this assumption, it was possible to correct for location guessing when an object was successfully recalled. This account, while not accounting explicitly for location swap errors, does seem reasonably appropriate given the small number of locations used $(\max =10)$, the large number of available locations to select from $(n=77)$, and the low incidence of location swap errors (mean $=0.69$ ). While the application of this guessing correction reduced the average value of $p(\mathrm{~L} \mid \mathrm{O})$ from 0.275 to $0.269(p<0.001)$, a reanalysis of the data showed no changes in the pattern or significance of the findings reported earlier.

In summary, correctly recalling the location of an object more effectively supported recalling the identity of the object at that location than vice versa (i.e. $p(\mathrm{O} \mid \mathrm{L})>p(\mathrm{~L} \mid \mathrm{O})$ ), and both conditional probabilities were higher for words than shapes. When participants successfully recalled locations, the probability of recalling the object at that location declined as the number of objects to remember increased. In contrast, when the participant successfully recalled an object, the probability of recalling the location of that object did not vary with memory load, except for when three words were presented when it was higher. Of particular importance, glucose tended to improve both conditional probabilities, and this indicated that it was equally effective in facilitating object recall (given correct location recall) and location recall (given correct object recall). Furthermore, this influence of glucose was not dependent on whether the objects were words or shapes or difficulty as indexed by the number of objects to remember.

\section{Blood glucose and object-location memory}

Our final analyses consider the influence of blood glucose measures on object-location memory. The trapezoid 
Table 3 Descriptive statistics for the conditional probabilities in the object-location memory task $( \pm$ $\mathrm{SE})$

\begin{tabular}{ccccccc}
\hline Conditional probability & Object type & Memory load & Glucose & \multicolumn{3}{c}{ Placebo } \\
\hline$p(\mathrm{O} \mid \mathrm{L})^{\mathrm{a}}$ & Words & 3 objects & 0.953 & $(0.030)$ & 0.919 & $(0.031)$ \\
& & 5 objects & 0.799 & $(0.050)$ & 0.594 & $(0.052)$ \\
& & 7 objects & 0.584 & $(0.043)$ & 0.590 & $(0.045)$ \\
& & 10 objects & 0.527 & $(0.045)$ & 0.428 & $(0.047)$ \\
& & 3 objects & 0.832 & $(0.039)$ & 0.862 & $(0.041)$ \\
& & 5 objects & 0.595 & $(0.060)$ & 0.596 & $(0.062)$ \\
& & 7 objects & 0.498 & $(0.050)$ & 0.463 & $(0.051)$ \\
& & 10 objects & 0.369 & $(0.037)$ & 0.333 & $(0.038)$ \\
& & 3 objects & 0.477 & $(0.038)$ & 0.401 & $(0.039)$ \\
& & 5 objects & 0.339 & $(0.032)$ & 0.237 & $(0.033)$ \\
& & 7 objects & 0.309 & $(0.036)$ & 0.294 & $(0.037)$ \\
& & 10 objects & 0.368 & $(0.042)$ & 0.272 & $(0.043)$ \\
& & 3 objects & 0.249 & $(0.031)$ & 0.244 & $(0.032)$ \\
& & 5 objects & 0.224 & $(0.033)$ & 0.219 & $(0.034)$ \\
& & 7 objects & 0.210 & $(0.030)$ & 0.180 & $(0.031)$ \\
& Shapes & 10 objects & 0.197 & $(0.028)$ & 0.172 & $(0.029)$ \\
& & & & & &
\end{tabular}

${ }^{\text {a }}$ Probability of recalling the object (given the location was correct)

${ }^{\mathrm{b}}$ Probability of recalling the location (given the object was correct) procedure described by Pruessner et al. (2003) was used to estimate two measures of regulation efficiency based on the area under the curve (AUC): AUC with respect to ground $\left(\mathrm{AUC}_{\mathrm{G}}\right.$ range $278-524 \mathrm{~min} \mathrm{mmol} / \mathrm{L}$ ) and $\mathrm{AUC}$ with respect to increase $\left(\mathrm{AUC}_{\mathrm{I}}\right.$ range $\left.37-240 \mathrm{~min} \mathrm{mmol} / \mathrm{L}\right)$. Using the following abbreviations-blood glucose at baseline $\left(\mathrm{BG}_{0}\right)$, mid-session $\left(\mathrm{BG}_{1}\right)$ and post-session $\left(\mathrm{BG}_{2}\right)$, and the time

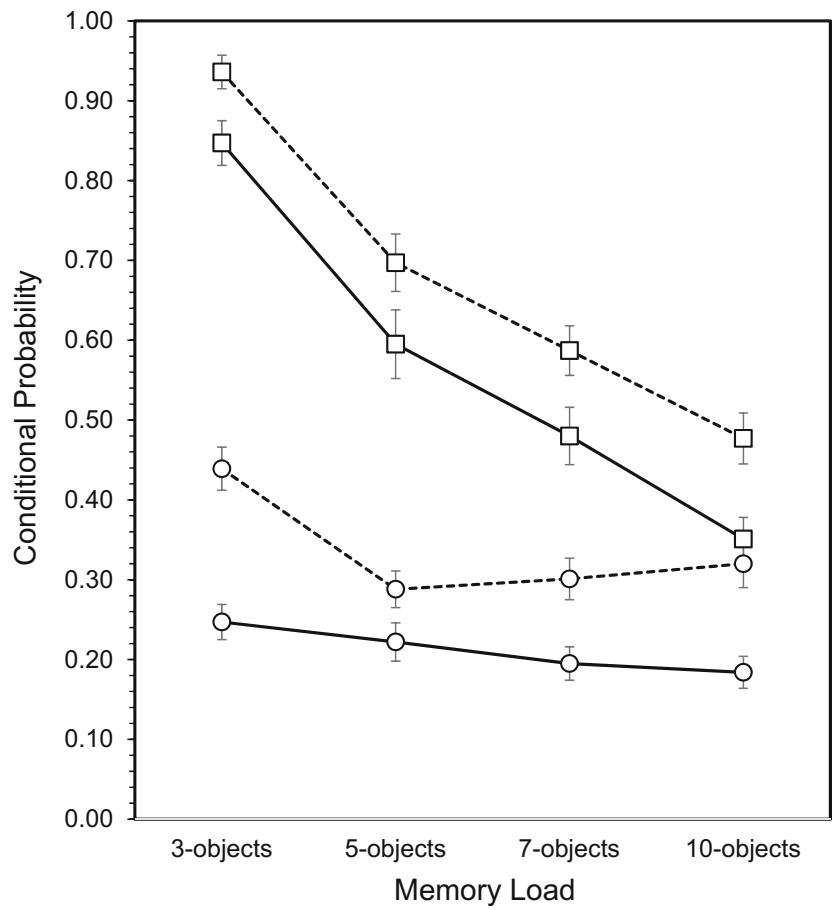

Fig. 4 Conditional probabilities as a function of object type and memory load $( \pm \mathrm{SE}) . \square p(\mathrm{O} \mid \mathrm{L}), \circ p(\mathrm{~L} \mid \mathrm{O})$. Dashed line words, solid line shapes interval (in minutes) between baseline and mid-session $\left(T_{01}\right)$, mid-session and post-session $\left(T_{12}\right)$, and baseline and postsession $\left(T_{02}\right)$-the two regulation indices are given by the following equations:

$$
\begin{gathered}
\left.\left.\mathrm{AUC}_{\mathrm{G}}=\left[\left(\mathrm{BG}_{0}+\mathrm{BG}_{1}\right) \times T_{01}\right) / 2\right]+\left[\left(\mathrm{BG}_{2}+\mathrm{BG}_{3}\right) \times T_{12}\right) / 2\right] \\
\mathrm{AUC}_{\mathrm{I}}=\mathrm{AUC}_{\mathrm{G}}-\left(\mathrm{BG}_{0} \times T_{02}\right) .
\end{gathered}
$$

Several authors point out that each measure provides unique information (e.g. Le Floch et al. 1990; Owen et al. 2013; Pruessner et al. 2003; Sünram-Lea et al. 2011), with $\mathrm{AUC}_{\mathrm{G}}$ taking into account fasting glucose levels in the total circulating glucose and $\mathrm{AUC}_{\mathrm{I}}$ the sensitivity of the system to the glucose load, irrespective of baseline glucose levels. For both measures, higher values are taken to indicate poorer regulation efficiency.

Table 4 displays the correlations between task performance and various measures of glucose, including $\mathrm{AUC}_{\mathrm{G}}$ and $\mathrm{AUC}_{\mathrm{I}}$. For the placebo group, the calculated values of $\mathrm{AUC}_{\mathrm{G}}$ (range 207-403 $\mathrm{min} \mathrm{mmol} / \mathrm{L}$ ) and $\mathrm{AUC}_{\mathrm{I}}$ (range -14 to $29 \mathrm{~min} \mathrm{mmol} /$ L) were also incorporated. Given the large difference between the two types of conditional probability, all three measures of conditional probability are shown (i.e. $p(\mathrm{O} \mid \mathrm{L}), p(\mathrm{~L} \mid \mathrm{O})$, and $p($ mean)) as are both forms of binding error. For completeness, correlations with matching variables (e.g. age, BMI) are also shown.

As Table 4 shows, demographic variables, mood, and baseline glucose levels were not correlated with any performance measure. As expected from the previous analyses, higher glucose levels were associated with better location memory, better binding memory, and higher conditional probabilities. The 
Table 4 Pearson correlations (two-tailed) between object-location memory, selected demographics, and blood glucose measures ( $n=31$ )

\begin{tabular}{|c|c|c|c|c|c|c|c|c|c|}
\hline & & & & & Conditi & al probabi & & Error type & \\
\hline & $\begin{array}{l}\text { Object } \\
\text { memory }\end{array}$ & $\begin{array}{l}\text { Location } \\
\text { memory }\end{array}$ & $\begin{array}{l}\text { Binding } \\
\text { memory }\end{array}$ & $\begin{array}{c}\text { Retrieval } \\
\text { Time }\end{array}$ & $p(\mathrm{O} \mid \mathrm{L})$ & $p(\mathrm{~L} \mid \mathrm{O})$ & $p($ mean $)$ & $\begin{array}{l}\text { Invalid } \\
\text { location }\end{array}$ & Location swap \\
\hline $\operatorname{Sex}^{\mathrm{a}}$ & 0.174 & 0.161 & 0.118 & -0.060 & -0.038 & 0.039 & -0.005 & 0.092 & 0.105 \\
\hline Age (years) & -0.211 & -0.210 & -0.165 & 0.038 & -0.078 & -0.094 & -0.104 & -0.071 & -0.196 \\
\hline BMI $\left(\mathrm{kg} / \mathrm{m}^{2}\right)$ & -0.012 & -0.328 & -0.280 & -0.047 & -0.120 & -0.301 & -0.245 & 0.174 & -0.185 \\
\hline Self-reported stress & 0.087 & 0.214 & 0.187 & -0.325 & 0.063 & -0.003 & 0.060 & -0.031 & 0.030 \\
\hline Self-reported arousal & 0.076 & -0.240 & -0.221 & -0.017 & -0.144 & -0.433 & -0.317 & 0.191 & 0.021 \\
\hline Task order ${ }^{\mathrm{a}}$ & -0.158 & -0.190 & -0.136 & -0.078 & 0.002 & -0.082 & -0.043 & -0.034 & -0.099 \\
\hline Glucose belief ${ }^{\mathrm{a}}$ & 0.073 & -0.150 & -0.086 & 0.009 & 0.178 & -0.030 & 0.107 & 0.090 & -0.152 \\
\hline Blood glucose: baseline & -0.156 & 0.103 & 0.101 & -0121 & -0.054 & 0.149 & 0.043 & -0.236 & 0.076 \\
\hline Blood glucose: average ${ }^{\mathrm{b}}$ & 0.117 & $0.453^{* *}$ & $0.571^{* *}$ & -0.132 & 0.317 & $0.475^{* *}$ & $0.475^{* *}$ & -0.160 & -0.016 \\
\hline Actual $\mathrm{AUC}_{\mathrm{G}}$ & -0.058 & $0.425^{*}$ & $0.537 * *$ & $0.469^{* *}$ & 0.331 & $0.566^{* *}$ & $0.534 * *$ & -0.265 & -0.035 \\
\hline Actual $\mathrm{AUC}_{\mathrm{I}}$ & -0.159 & $0.415^{*}$ & $0.559 * *$ & 0.013 & $0.383 *$ & $0.458 * *$ & $0.511 * *$ & -0.080 & -0.083 \\
\hline
\end{tabular}

${ }^{\text {a }}$ Point biserial correlation

${ }^{\mathrm{b}}$ Average of mid- and post-session

${ }^{*} p<0.05 ; * *<0.01$

same pattern was seen for the two measures of regulation efficiency, with poorer glucose regulation being associated with better performance. There are two points of interest to note. First, when the two types of conditional probability were separated, the correlations were stronger for $p(\mathrm{~L} \mid \mathrm{O})$ than for $p(\mathrm{O} \mid \mathrm{L})$. Specifically, for $p(\mathrm{~L} \mid \mathrm{O})$ the correlations were significant, but for $p(\mathrm{O} \mid \mathrm{L})$, correlations with blood glucose $(p=$ $0.082)$ and $\mathrm{AUC}_{\mathrm{G}}(p=0.069)$ only showed trends. Second, the correlation between retrieval time and $\mathrm{AUC}_{\mathrm{G}}$ suggests that poorer regulators take longer to complete the retrieval phase.

To examine this further, Table 5 shows the correlations within the glucose and placebo groups. For the glucose group, higher mid-session blood glucose was associated with better location memory $(r(14)=0.551, p=0.027)$, better binding memory $(r(14)=0.609, p=0.012)$, and higher conditional probabilities $(r(14)=0.558, p=0.025)$. Again, the correlations were stronger for $p(\mathrm{~L} \mid \mathrm{O})$ than $p(\mathrm{O} \mid \mathrm{L}), r(14)=0.755, p=0.001$ and $r(14)=0.288, p=0.279$, respectively, and the difference between these two correlations was significant $(z=2.11, p=$ 0.034; see Fig. 5). Changes in blood glucose from the mid- to post-session period were not associated with any performance measure. For $\mathrm{AUC}_{\mathrm{G}}$ and $\mathrm{AUC}_{\mathrm{I}}$ respectively, there were no significant correlations with location memory $(r(14)=0.218$, $p=0.416$ and $r(14)=0.260, p=0.331)$, only weak effects for binding memory $(r(14)=0.380, p=0.146$ and $r(14)=0.444$, $p=0.085)$, but a stronger relationship with conditional probabilities $(r(14)=0.512, p=0.043$ and $r(14)=0.597, p=0.015)$. Again, while the correlations were stronger for $p(\mathrm{~L} \mid \mathrm{O})$ than $p(\mathrm{O} \mid \mathrm{L})$, the difference between the two correlations was not significant for either $\mathrm{AUC}_{\mathrm{G}}(z=0.704, p=0.482)$ or $\mathrm{AUC}_{\mathrm{I}}(z=$ $0.603, p=0.547)$. Finally, as with the earlier analysis, higher $\mathrm{AUC}_{\mathrm{G}}$ values were associated with longer retrieval times $(r(14)=0.611, p=0.012)$. Interestingly, within the placebo group, only the correlation between $\mathrm{AUC}_{\mathrm{G}}$ and retrieval times $(r(13)=0.831, p<0.001)$ was significant.

The observation that higher $\mathrm{AUC}_{\mathrm{G}}$ values were correlated with longer retrieval times in both the glucose and placebo groups warrants closer inspection. The calculation of $\mathrm{AUC}_{\mathrm{G}}$ incorporates the time interval between blood glucose measures (see Pruessner et al. 2003) and this interval is, in turn, determined by individual differences in the average recall time on this self-paced task. Indeed, the correlation between total session length and average recall time $(r(29)=0.916$, $p<0.001)$ simply indicates that the time interval between blood glucose measures was longer for slower participants. As longer intervals between glucose measurements increase AUC values, there exists some confounding between AUC values and average recall time.

In an effort to disentangle individual variations in session duration from individual variations in AUC measures, an attempt was made to normalize AUC scores, operationalized by dividing an individual's AUC score by their session length (in minutes) and then pro-rating this value to the average session length $(53 \mathrm{~min})$. It is important to note that there were no differences in the relative timing of blood glucose measurements for the glucose and placebo groups. We applied this normalization procedure to $\mathrm{AUC}_{\mathrm{G}}$ and $\mathrm{AUC}_{\mathrm{I}}$ because both incorporate the time interval between blood glucose measurements. As shown in Table 5, using these normalized measures, the correlation between $\mathrm{AUC}_{\mathrm{G}}$ and recall time was no longer significant for either the glucose $(r(14)=-0.077, p=0.778)$ or the placebo $(r(13)=0.013, p=0.962)$ group. Thus, for the placebo group, no significant associations remained between glucose measures and task performance. In contrast, for the 
Table 5 Pearson correlations (two-tailed) between object-location memory and within group measures of blood glucose

\begin{tabular}{|c|c|c|c|c|c|c|c|c|c|}
\hline & & & & & Conditic & al probabi & & Error type & \\
\hline & $\begin{array}{l}\text { Object } \\
\text { memory }\end{array}$ & $\begin{array}{l}\text { Location } \\
\text { memory }\end{array}$ & $\begin{array}{l}\text { Binding } \\
\text { memory }\end{array}$ & $\begin{array}{l}\text { Retrieval } \\
\text { time }\end{array}$ & $p(\mathrm{O} \mid \mathrm{L})$ & $p(\mathrm{~L} \mid \mathrm{O})$ & $p($ mean $)$ & $\begin{array}{l}\text { Invalid } 1 \\
\text { ocation }\end{array}$ & $\begin{array}{c}\text { Location } \\
\text { swap }\end{array}$ \\
\hline \multicolumn{10}{|l|}{ Glucose group $(n=16)$} \\
\hline Blood glucose: baseline & -0.166 & 0.214 & 0.075 & -0.294 & -0.168 & 0.156 & -0.048 & -0.348 & 0.310 \\
\hline Blood glucose: mid-session & -0.145 & $0.551 *$ & $0.609 *$ & 0.075 & 0.288 & $0.755^{* *}$ & $0.558^{*}$ & $-0.468 \#$ & -0.047 \\
\hline Blood glucose: change $^{\mathrm{a}}$ & -0.167 & -0.155 & -0.170 & -0.289 & 0.016 & -0.100 & -0.035 & -0.050 & -0.033 \\
\hline Actual $\mathrm{AUC}_{\mathrm{G}}$ & -0.167 & 0.218 & 0.380 & $0.611 *$ & 0.362 & $0.542 *$ & $0.512 *$ & -0.242 & -0.227 \\
\hline Normalized $\mathrm{AUC}_{\mathrm{G}}$ & -0.234 & 0.425 & $0.453 \#$ & -0.077 & 0.237 & $0.641 * *$ & $0.468 \#$ & $-0.481 \#$ & -0.020 \\
\hline Actual $\mathrm{AUC}_{\mathrm{I}}$ & -0.117 & 0.260 & $0.444 \#$ & 0.359 & $0.449 \#$ & $0.594 *$ & $0.597 *$ & -0.211 & -0.324 \\
\hline Normalized $\mathrm{AUC}_{\mathrm{I}}$ & -0.146 & 0.332 & $0.470 \#$ & 0.133 & 0.402 & $0.628 * *$ & $0.580^{*}$ & -0.295 & -0.257 \\
\hline \multicolumn{10}{|l|}{ Placebo group $(n=15)$} \\
\hline Blood glucose: baseline & -0.275 & -0.167 & -0.045 & 0.138 & 0.022 & 0.062 & 0.054 & -0.179 & -0.232 \\
\hline Blood glucose: average ${ }^{b}$ & -0.261 & -0.123 & -0.058 & -0.014 & 0.006 & 0.040 & 0.030 & -0.175 & -0.187 \\
\hline Actual $\mathrm{AUC}_{\mathrm{G}}$ & -0.399 & 0.309 & 0.298 & $0.831^{* *}$ & 0.034 & 0.475 & 0.325 & -0.435 & 0.103 \\
\hline Normalized $\mathrm{AUC}_{\mathrm{G}}$ & -0.322 & -0.144 & -0.081 & 0.013 & -0.040 & 0.040 & -0.003 & -0.217 & -0.187 \\
\hline Actual $\mathrm{AUC}_{\mathrm{I}}$ & -0.073 & 0.096 & -0.062 & -0.162 & -0.150 & -0.033 & -0.127 & -0.072 & 0.151 \\
\hline Normalized $\mathrm{AUC}_{\mathrm{I}}$ & -0.043 & 0.070 & -0.059 & -0.256 & -0.119 & -0.051 & -0.116 & -0.042 & 0.123 \\
\hline
\end{tabular}

${ }^{a}$ Mid- to post-session increase

${ }^{\mathrm{b}}$ Average of mid- and post-session

${ }^{*} p<0.05 ; * * p<0.01 ; \# p<0.10$

glucose group, the other performance associations with $\mathrm{AUC}_{\mathrm{G}}$ and $\mathrm{AUC}_{\mathrm{I}}$ were robust to this normalization.

In summary, for the glucose group, higher measures of glucose were related to better performance on the objectlocation task. The most compelling of these were the positive associations with object-location binding memory and $p(\mathrm{~L} \mid \mathrm{O})$. In contrast, for the placebo group, there were no significant

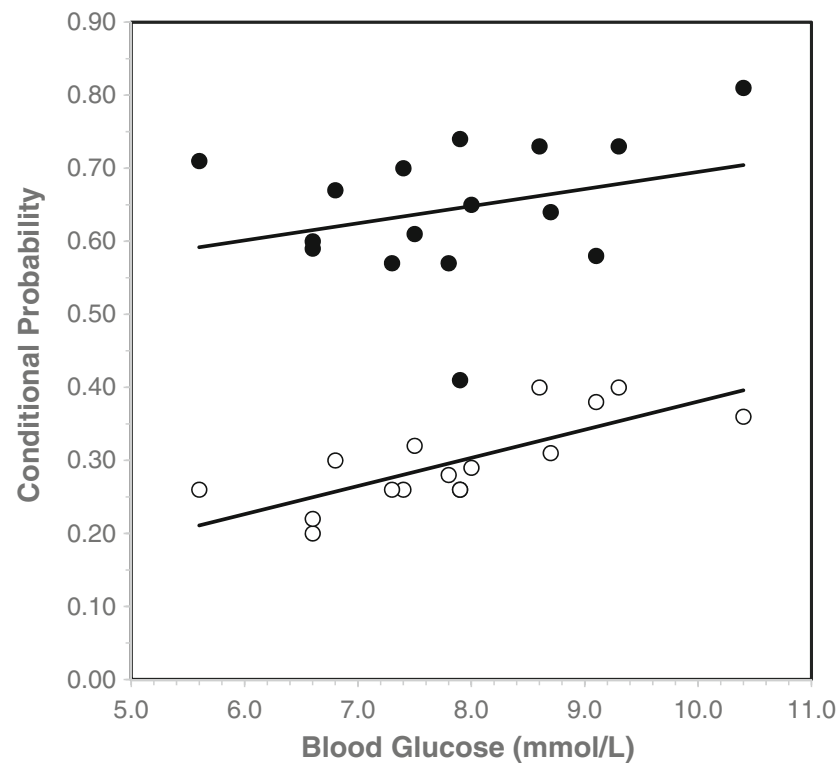

Fig. 5 Linear regression lines showing the correlation between the two conditional probability measures and mid-session blood glucose levels within the glucose group. $\bullet p(\mathrm{O} \mid L), \circ p(L \mid \mathrm{O})$ glucose correlations with object-location performance. The initial observation that higher $\mathrm{AUC}_{\mathrm{G}}$ values were associated with slower recall times in both the placebo and glucose groups was likely to result from the integration of the time between glucose measurements into AUC measures because these associations were eliminated once the AUC measures were normalized for session duration.

\section{Discussion}

The current study examined the influence of a 30-g glucose drink on object-location memory. Participants viewed a variable number of objects (words or shapes) in different locations and immediately attempted to reproduce the objects in their remembered locations. Our basic findings indicate that glucose consumption improves the ability to remember the locations of objects and to successfully bind objects to their location. Furthermore, while increasing the number of objects to remember produces a detrimental effect on recalling the objects and on how successfully those objects were bound to locations, the beneficial influence of glucose remained stable across this difficulty manipulation.

Many researchers have noted that the binding objects to locations requires communication between the ventral (what) and dorsal (where) anatomical processing streams (Bentley and Salinas 2009; Eichenbaum et al. 2012) and on the basis of previous research on object-location memory (e.g. Crane 
and Milner 2005; Gilbert and Kesner 2004; Hannula and Ranganath 2008; Olson et al. 2006; Piekema et al. 2006; Postma et al. 2008), the preferential impact of glucose on binding memory is consistent with a hippocampal influence. Indeed, as the hippocampal system receives converging information from several association cortices (Shastri 2002), it is well suited to the fundamental process of binding diverse aspects of an experience into a unified episodic representation. The two principal theories of hippocampal function concern its involvement in the creation of episodic memories (e.g. Squire 1992) and its importance for spatial cognition (e.g. O'Keefe and Nadel 1978). Reconciliation of these two conceptions has been proposed (Eichenbaum 1999; Eichenbaum and Cohen Neal 2014), and the object-location task used here explicitly exploits both the episodic and spatial functions. In addition to enhancing the binding of object-location pairs, we found that glucose exerts a stronger influence on location memory than on object memory, suggesting a special role for glucose in enhancing human spatial memory. This is consistent with the body of human work on spatial cognition and the hippocampus (see Hartley et al. 2014) and parallel observations in rodents of both hippocampal contributions to spatial memory (Eichenbaum 1999; Geva-Sagiv et al. 2015; Mumby et al. 2002; Squire 1992) and improvements of spatial memory with glucose (Dash et al. 2006; McNay et al. 2000; Winocur and Gagnon 1998).

Following the creation of a bound representation comprising location and identity attributes, the retrieval of one attribute acts as a retrieval cue for the other. Although this reciprocal cueing could be symmetrical, our conditional probability analysis shows that the temporary binding created is strongly asymmetric. Specifically, remembering a location more effectively aids recalling the object at that location than remembering an object aids recalling the location of that object. This asymmetry accords well with past research showing that location has a special status in the processing of objects (e.g. Pertzov and Husain 2014; Rajsic and Wilson 2014; Roth and Franconeri 2012). The other work has shown that attending to specific locations can initiate object processing at that location (Treisman and Zhang 2006) and while the object's location seems to be automatically encoded when attention is directed to non-spatial attributes (e.g. colour), these nonspatial attributes are not necessarily encoded when attention is simply drawn to the location (e.g. Chen 2009; Golomb et al. 2014a). Similarly, in change detection tasks, the memory for object identity is impaired by changing the locations of objects, but memory for location is unaffected by changes in object identity (Jiang et al. 2000, 2004; Poch et al. 2010). Therefore, one way of understanding this asymmetric binding strength within object-location pairs is that location provides the nucleus for the binding process. Our memory for where an object was seen is strongly bound to the memory about what object was seen there. In contrast, our memory for what the object was is only weakly bound to where it was seen. Other authors have noted the pervasiveness of such processing asymmetries in the visual system, presumably because an object must occupy a specific spatial location, whereas it is not necessary for space to contain an object (e.g. Chen 2009). It is important to note that this asymmetric binding is common to those who consumed placebo and glucose, and there is no compelling evidence that this basic asymmetry is altered by consuming glucose. Rather, glucose simply appears to strengthen the probability of gaining access to each attribute of the bound pair, given memory for the other attribute.

In connection with this asymmetry, we also found superior location memory and object-location binding, for words compared to shapes. One basis for this advantage could be the rich semantic connections of words, compared to shapes, since this creates greater contextual detail, thereby supporting retrieval. However, this advantage would also be expected to improve object memory and there is no evidence for this except at low memory loads. The most likely source is that each word is only used once, but each shape is used six times. This renders word-location bindings unique because each word had never appeared anywhere else. In contrast, shape-location bindings are not unique because when a shape is repeated, it needs to be bound to a new location. This differential mapping seems to render shape-location binding less stable (e.g. enduring bindings hindering the creation of new ones). If, as suggested above, location provides the nucleus for the binding process, then this is consistent with the observations that both types of conditional probability are lower for shapes, that location memory is poorer for shapes, and location-based errors are more common for shapes. Finally, the asymmetric cueing between object and location is preserved across both shapes and words for all memory loads, with one notable exception: the three-word condition for $p(\mathrm{~L} \mid \mathrm{O})$. For this condition, memory for words, locations, and bindings are especially high and the outcome is a selective enhancement on remembering where a correctly recalled word was. Again, this points to a central role for location in the binding process, but at the lowest memory load greater accuracy concerning where a remembered word was seen (e.g. via retrieval of semantic detail). In any case, whatever processes underlie differences in shape-location and word-location binding across the different memory loads, the current study found no evidence that glucose modifies those processes.

While the evidence above indicates that glucose simply strengthens the temporary bond between location and identity attributes irrespective of the bond direction and type of object, the analysis of blood glucose variations suggests a more nuanced picture. Across all participants, baseline glucose levels were not correlated with any aspects of performance, but higher levels following glucose consumption were associated with better location memory, object-location binding memory, and higher average conditional probabilities. Critically, a 
separation of the two conditional probabilities indicated that higher blood glucose, and the two measures of area under the curve, tended to be more reliably correlated with the weaker of the two bonds: $p(\mathrm{~L} \mid \mathrm{O})$, retrieving the location of a successfully recalled object. This pattern was only seen within the glucose group. Furthermore, this pattern is consistent with the glucose effect size being (non-significantly) stronger for $p(\mathrm{~L} \mid \mathrm{O})$ than $p(\mathrm{O} \mid \mathrm{L}): d=0.654$ vs. 0.508 . This raises the intriguing possibility that higher glucose levels may preferentially strengthen the weaker of the two bonds within object-location pairs. That is, an effect of task complexity with glucose having a stronger impact on the more difficult component. The question therefore is how difficulty or complexity should be conceived in this situation, and to understand this we must turn to the respective roles of object and location information. The features that identify an object rarely change over time, but this is not true for the location of that object. It follows that binding an object to a location is always temporary and in normal circumstances the link needs continual updating. The relative weakness of this link may be an advantage, permitting the required flexibility for unbinding, renewing, or updating the link between the object and its current location. Knowing what an object was does not pose this updating problem. Consistent with this idea, our data shows access to what knowledge declines rapidly as memory load increases, but access to where knowledge is not influenced by memory load. For the conditional probabilities, access to objects at remembered locations is subject to this memory load effect, but access to locations from remembered objects is not. Thus, the enhancement of this weaker bond by glucose may indicate that glucose plays a preferential role in adaptably binding an object to its current location. Thus, difficulty could be broadly conceived as relating to the differential demands of flexibly updating information.

However, given the small sample sizes, it would be premature to infer too much from the different strengths of the glucose relationships with the two conditional probabilities. The main evidence suggests that glucose improves both bonds equivalently, rather than one more than another. Based on our correlational analysis, for those given glucose, we simply find that higher glucose levels are associated with better binding memory and conditional probability scores. These positive correlations may also be interpreted as reflecting the influence of glucose regulation efficiency, with poor regulators showing the most improvement. The selective strengthening of the weaker of the two bonds linking object and location, however, must remain tentative. This is not simply because only one glucose measure shows a significant difference in correlations but also because an interactive influence of glucose on the two conditional probabilities was not detected in the main analysis. Additionally, the improvement in location memory following glucose consumption is clearly independent of blood glucose variations within the glucose group.
There are a number of potential process, discussed below, that could underlie the strengthening of the bond between objects and locations. Further work is clearly required for a more complete understanding of how this strengthening occurs, but it seems likely that enhanced location memory will occupy a central role. While early models proposed that attention binds or "glues" different features of objects together (Treisman 1988), simple objects comprising intrinsic combinations of single features (e.g. blue circle) are not attention demanding, being processed automatically prior to entering the episodic buffer of working memory (Allen et al. 2012). The binding of objects to locations is also thought to occur automatically (Chen and Wyble 2015; Treisman and Zhang 2006) but appears particularly fragile because delays of several seconds can disrupt this binding, particularly with highmemory loads (Pertzov et al. 2012). This disruption is not due to failures in remembering objects, but declines in the precision of remembering locations, pointing to difficulties in maintaining bound representations (see Olson et al. 2006). Other work has shown that maintaining bindings involving location produces sustained activation of the hippocampus, whereas maintaining non-spatial bindings does not (Piekema et al. 2006). Consistent with this are the deficits in objectlocation memory for patients with hippocampal damage (Hampstead et al. 2011; Hannula and Ranganath 2008; Hannula et al. 2006; Yee et al. 2014), with the prefrontal cortex supporting both the encoding (Spellman et al. 2015) and the maintenance (Campo et al. 2005; Prabhakaran et al. 2000 ) of spatial information in working memory. Contrary to this evidence, Allen et al. (2014) report preserved colourlocation binding for up to $10 \mathrm{~s}$ in a patient with selective loss of hippocampal tissue. However, importantly, binding fell to chance levels when assessed only a few minutes later. Thus, while it remains plausible that glucose promotes the formation of object-location bindings, the above observations suggest that their fragility lies in maintaining them and this seems the most likely source of the glucose facilitation observed.

One important function of the episodic buffer, which acts as an interface between working memory and longer-term episodic memory, is maintaining integrated memory representations (Baddeley et al. 2011). Whether the hippocampus can feasibly represent the anatomical site linking working and longer-term episodic memory remains unresolved (see Baddeley et al. 2011 for a discussion), but if glucose supports memory maintenance, then several bases for the enhanced binding are possible. Initially, it would seem sensible to establish that improved binding due to glucose is restricted to tasks with a spatial basis. A comparison of non-spatial (e.g. colourshape) and spatial (e.g. colour-location) bindings would determine whether glucose operates selectively on tasks with a spatial component or more generally on binding processes. This is important because the hippocampal system supports a wide range of binding processes (Olsen et al. 2012) and 
while there is some evidence that glucose does not enhance the short-term maintenance of non-spatial (word-word) bindings (Stollery and Christian 2015), it would seem prudent to establish the proposed role of spatial memory in the observed enhancement. Thus, the possibility that spatial processing is an important feature of binding tasks that show short-term enhancement due to glucose remains to be determined. Additionally, examining binding efficiency changes over time would supply evidence relevant to the proposal that improved maintenance (e.g. slower decay rates or improved resistance to interference) underlies the improvement.

Several studies have reported evidence consistent with glucose producing a short-term increase in the efficient use of limited attentional resources (Benton et al. 1987, 1994; Flint and Turek 2003; Fucetola et al. 1999; Rao et al. 2005; Riby et al. 2008; Scholey et al. 2009; Serra-Grabulosa et al. 2010) and, given participants were explicitly required to remember object-location pairings, this could be one possible mechanism for improved binding. Specifically, our task requires the maintenance of multiple object-location pairs and participants may have strategically attended to a subset of these pairs by prioritizing resources. The impact of glucose presumably relates to locations because there are no reliable differences in the retention of the objects themselves. Under this scenario, there are two possible, but not mutually exclusive, processes whereby glucose could improve maintenance. The first is that glucose permits the active maintenance of a larger subset of locations. The second is that the subset of locations not actively maintained is less subject to degradation with glucose. Both processes permit improved binding efficiency, particularly if locations provide the binding nucleus. However, it remains unclear why glucose improvements should be equivalent for three object-location pairings, which should not tax attentional resources, and ten object-location pairings, which would. As such, therefore, this suggestion requires further scrutiny.

In connection with this, maintaining integrated information in the episodic buffer is also dependent on attentional resources (Baddeley et al. 2011), and reduced binding effectiveness of letter-location pairs is seen when a concurrent memory load is retained over a brief period (Elsley and Parmentier 2009). Even without a concurrent load, letter-location bindings decline rapidly during the first $5 \mathrm{~s}$ and then stabilize for at least another $10 \mathrm{~s}$ (Elsley and Parmentier 2015). These data suggest that attentional resources are critical for short-term maintenance. For comparison, the average reproduction times in our study ranged from $17 \mathrm{~s}$ (three items) to $37 \mathrm{~s} \mathrm{(ten} \mathrm{items),}$ making even our lowest memory load beyond this short-term maintenance period.

Other possibilities invoke known hippocampal functions. While the object-location task used here does not involve the complex spatial processes required for navigating environments, participants must utilize some form of egocentric or allocentric processing to retain the spatial layout of objects.
While it is known that the hippocampus primarily supports allocentric processing (O'Keefe and Nadel 1978), both can cooperate to support performance (Burgess 2006; Ekstrom et al. 2014). In our study, egocentric processing would encode each object-location pair from the participant's viewpoint. Allocentric processing would encode object-location pairs in relation to each other and establish a higher-order representation incorporating the configuration of locations. Finally, when the participant recalls the material, they must reorient from the vertical presentation format to the horizontal recall format. This description provides two possible mechanisms for how glucose might improve location memory and binding. First, transferring from the vertical presentation phase to the horizontal recall phase initiates attentional-updating process that can disrupt temporary object-location bindings (Golomb et al. 2014b). Thus, the superior binding following glucose may indicate a memory representation that is more resistant to disruption. Alternatively, preferential allocentric-based processing of the spatial array may create a memory representation that better preserves the configural properties of the display, with this viewpoint-independent representation better supporting location memory and, consequently, binding. Of course, since our participants were explicitly required to remember what objects went where and we tested memory using free-recall, rather than recognition, the conscious controlled recollection that this entails could provide another hippocampal-based process capable of sustaining binding. In this context, it is important to note that while location encoding may be automatic, the later retrieval of that location is clearly not. Given that recollective experiences are associated with more contextual knowledge and location appears to be a strongly encoded contextual feature when memorizing objects (Perfect et al. 1996), the importance of the hippocampal system to the encoding and retrieval of contextual features (e.g. location) could form a general basis for the findings and is consistent with other studies showing better recollection with glucose (e.g. Smith et al. 2009; Sunram-Lea et al. 2008).

As noted earlier, the hippocampal system lies at the centre of the core function of binding disparate elements together, and it is known that object-location memory shows complex cooperative interactions among several brain regions such as the hippocampus, perirhinal cortex, and the medial prefrontal cortex (see Barker et al. 2007; Barker and Warburton 2009, 2015; Warburton and Brown 2010). For example, there is good anatomical evidence for projections between the hippocampus and medial prefrontal cortex, medial prefrontal cortex and perirhinal cortex, and perirhinal cortex, via the entorhinal cortex, to the hippocampus (e.g. Brown and Aggleton 2001). Furthermore, lesions of the parahippocampal and fornix regions, two important hippocampal communication pathways, impair object-location memory (Bussey et al. 2000). More importantly for a neurochemical understanding of how glucose might improve performance, there seems to be a critical 
role for the cholinergic system within this circuit for objectlocation memory (Barker and Warburton 2009). Other work on object-location memory in rats has also shown that NMDA receptor antagonists and $\mathrm{mACh}$ antagonists impair memory, NMDA agonist and cholinesterase inhibitors improve memory, and inactivation of the CA1 hippocampal area blocks learning (Assini et al. 2009). These observations are consistent with one of the several possible mechanisms whereby glucose modulates episodic memory, that of facilitating acetylcholine synthesis (Messier 2004; Riby and Riby 2006).

In conclusion, the current study found that glucose improves the ability to remember the locations of objects and to bind objects to those locations. Based on current knowledge, this suggests a strong hippocampal basis and the findings are therefore consistent with the view that glucose has an affinity for hippocampal-based cognitive processing. How best to understand these improvements, and the extent spatial processing plays a critical role, requires further exploration as several suggestions are compatible with the current data. The asymmetric binding strength of the two bonds linking object and location, together with the notion that glucose might selectively enhance the updating of contextual detail, is clearly in need of further investigation. Nevertheless, our study highlights the usefulness of the object-location paradigm for progressing our understanding of the functional basis for glucose improvements in cognition. This is especially pertinent given that the improvements we found differentiate, using a single paradigm, three fundamental aspects of episodic memory: the "what", the "where", and the "what-was-where".

Open Access This article is distributed under the terms of the Creative Commons Attribution 4.0 International License (http:// creativecommons.org/licenses/by/4.0/), which permits unrestricted use, distribution, and reproduction in any medium, provided you give appropriate credit to the original author(s) and the source, provide a link to the Creative Commons license, and indicate if changes were made.

\section{References}

Aggleton JP (2012) Multiple anatomical systems embedded within the primate medial temporal lobe: implications for hippocampal function. Neurosci Biobehav Rev 36:1579-1596. doi:10.1016/j. neubiorev.2011.09.005

Allen RJ, Hitch GJ, Mate J, Baddeley AD (2012) Feature binding and attention in working memory: a resolution of previous contradictory findings. Q J Exp Psychol 65:2369-2383. doi:10.1080/17470218. 2012.687384

Allen RJ, Vargha-Khadem F, Baddeley AD (2014) Item-location binding in working memory: Is it hippocampus-dependent? Neuropsychologia 59:74-84. doi:10.1016/j.neuropsychologia. 2014.04.013

Assini FL, Duzzioni M, Takahashi RN (2009) Object location memory in mice: pharmacological validation and further evidence of hippocampal CA1 participation. Behav Brain Res 204:206-211. doi:10.1016/ j.bbr.2009.06.005
Bachevalier J, Nemanic S (2008) Memory for spatial location and objectplace associations are differently processed by the hippocampal formation, parahippocampal areas TH/TF and perirhinal cortex. Hippocampus 18:64-80. doi:10.1002/hipo.20369

Baddeley AD, Allen RJ, Hitch GJ (2011) Binding in visual working memory: the role of the episodic buffer. Neuropsychologia 49: 1393-1400. doi:10.1016/j.neuropsychologia.2010.12.042

Barker GRI, Warburton EC (2009) Critical role of the cholinergic system for object-in-place associative recognition memory. Learn Mem 16: 8-11. doi:10.1101/lm.1121309

Barker GRI, Warburton EC (2015) Object-in-place associative recognition memory depends on glutamate receptor neurotransmission within two defined hippocampal-cortical circuits: a critical role for AMPA and NMDA receptors in the hippocampus, perirhinal, and prefrontal cortices. Cereb Cortex 25:472-481. doi:10.1093/cercor/bht245

Barker GRI, Bird F, Alexander V, Warburton EC (2007) Recognition memory for objects, place, and temporal order: a disconnection analysis of the role of the medial prefrontal cortex and perirhinal cortex. J Neurosci 27:2948-2957. doi:10.1523/jneurosci.5289-06.2007

Bentley NM, Salinas E (2009) Neural coding of spatial representations. In: Squire LR (ed) Encyclopedia of neuroscience. Academic, New York, pp 117-122

Benton D, Owens DS (1993) Blood glucose and human memory. Psychopharmacology 113:83-88. doi:10.1007/BF02244338

Benton D, Parker PY (1998) Breakfast, blood glucose, and cognition. Am J Clin Nutr 67:772S-778S

Benton D, Sargent J (1992) Breakfast, blood glucose and memory. Biol Psychol 33:207-210. doi:10.1016/0301-0511(92)90032-P

Benton D, Stevens MK (2008) The influence of a glucose containing drink on the behavior of children in school. Biol Psychol 78:242245. doi:10.1016/j.biopsycho.2008.03.007

Benton D, Brett V, Brain PF (1987) Glucose improves attention and reaction to frustration in children. Biol Psychol 24:95-100. doi:10. 1016/0301-0511(87)90016-0

Benton D, Owens DS, Parker PY (1994) Blood glucose influences memory and attention in young adults. Neuropsychologia 32:595-607. doi:10.1016/0028-3932(94)90147-3

Berch DB, Krikorian R, Huha EM (1998) The Corsi block-tapping task: methodological and theoretical considerations. Brain Cogn 38:317338. doi:10.1006/brcg.1998.1039

Brown MW, Aggleton JP (2001) Recognition memory: What are the roles of the perirhinal cortex and hippocampus? Nat Rev Neurosci 2:5161. doi: $10.1038 / 35049064$

Brunetti R, Del Gatto C, Delogu F (2014) eCorsi: implementation and testing of the Corsi block-tapping task for digital tablets. Front Psychol 5:939. doi:10.3389/fpsyg.2014.00939

Burgess N (2006) Spatial memory: how egocentric and allocentric combine. Trends Cogn Sci 10:551-557. doi:10.1016/j.tics.2006.10.005

Bussey TJ, Duck J, Muir JL, Aggleton JP (2000) Distinct patterns of behavioural impairments resulting from fornix transection or neurotoxic lesions of the perirhinal and postrhinal cortices in the rat. Behav Brain Res 111:187-202. doi:10.1016/S0166-4328(00)00155-8

Campo P, Maestú F, Ortiz T, Capilla A, Santiuste M, Fernández A, Amo C (2005) Time modulated prefrontal and parietal activity during the maintenance of integrated information as revealed by magnetoencephalography. Cereb Cortex 15:123-130. doi:10.1093/cercor/bhh115

Caprio M, Godoy JPMC, Galera C (2010) Binding spatial location and stimulus identity in short-term memory. Psychol Neurosci 3:125132. doi:10.3922/j.psns.2010.1.016

Chen Z (2009) Not all features are created equal: processing asymmetries between location and object features. Vision Res 49:1481-1491. doi: $10.1016 / j$.visres.2009.03.008

Chen H, Wyble B (2015) The location but not the attributes of visual cues are automatically encoded into working memory. Vision Res 107: 76-85. doi:10.1016/j.visres.2014.11.010 
Cohen NJ, Ryan J, Hunt C, Romine L, Wszalek T, Nash C (1999) Hippocampal system and declarative (relational) memory: summarizing the data from functional neuroimaging studies. Hippocampus 9:83-98. doi:10.1002/(sici)1098-1063(1999)9:1<83::aid-hipo9>3. $0 . \operatorname{co} ; 2-7$

Crane J, Milner B (2005) What went where? Impaired object-location learning in patients with right hippocampal lesions. Hippocampus 15:216-231. doi:10.1002/hipo.20043

Dash PK, Orsi SA, Moore AN (2006) Spatial memory formation and memory-enhancing effect of glucose involves activation of the tuberous sclerosis complex-mammalian target of rapamycin pathway. J Neurosci 26:8048-8056. doi:10.1523/JNEUROSCI.0671-06. 2006

Dennis SH, Jaafari N, Cimarosti H, Hanley JG, Henley JM, Mellor JR (2011) Oxygen/glucose deprivation induces a reduction in synaptic AMPA receptors on hippocampal CA3 neurons mediated by mGluR1 and adenosine A3 receptors. J Neurosci 31:11941-11952. doi:10.1523/jneurosci.1183-11.2011

Dent K, Smyth MM (2005) Verbal coding and the storage of formposition associations in visual-spatial short-term memory. Acta Psychologica 120:113-140. doi:10.1016/j.actpsy.2005.03.004

Eichenbaum H (1999) The hippocampus and mechanisms of declarative memory. Behav Brain Res 103:123-133. doi:10.1016/S01664328(99)00044-3

Eichenbaum H, Cohen Neal J (2014) Can we reconcile the declarative memory and spatial navigation views on hippocampal function? Neuron 83:764-770. doi:10.1016/j.neuron.2014.07.032

Eichenbaum H, Sauvage M, Fortin N, Komorowski R, Lipton P (2012) Towards a functional organization of episodic memory in the medial temporal lobe. Neurosci Biobehav Rev 36:1597-1608. doi:10.1016/ j.neubiorev.2011.07.006

Ekstrom AD, Arnold AEGF, Iaria G (2014) A critical review of the allocentric spatial representation and its neural underpinnings: toward a network-based perspective. Front Hum Neurosci 8:803. doi:10.3389/fnhum.2014.00803

Elsley JV, Parmentier FBR (2009) Is verbal-spatial binding in working memory impaired by a concurrent memory load? Q J Exp Psychol 62:1696-1705. doi:10.1080/17470210902811231

Elsley JV, Parmentier FBR (2015) The asymmetry and temporal dynamics of incidental letter-location bindings in working memory. Q J Exp Psychol 68:433-441. doi:10.1080/17470218.2014.982137

Faul F, Erdfelder E, Lang A-G, Buchner A (2007) G*Power 3: a flexible statistical power analysis program for the social, behavioral, and biomedical sciences. Behav Res Methods 39:175-191. doi:10. 3758/BF03193146

Fearnley S (1997) MRC psycholinguistic database search program. Behav Res Methods Instrum Comput 29:291-295. doi:10.3758/ BF03204829

Finke C, Braun M, Ostendorf F, Lehmann T-N, Hoffmann K-T, Kopp U, Ploner CJ (2008) The human hippocampal formation mediates short-term memory of colour-location associations. Neuropsychologia 46:614-623. doi:10.1016/j.neuropsychologia. 2007.10.004

Flint RW Jr, Turek C (2003) Glucose effects on a continuous performance test of attention in adults. Behav Brain Res 142:217-228. doi:10. 1016/S0166-4328(03)00002-0

Fucetola R, Newcomer JW, Craft S, Melson AK (1999) Age and dosedependent glucose-induced increases in memory and attention in schizophrenia. Psychiatry Res 88:1-13. doi:10.1016/S01651781(99)00063-3

Geva-Sagiv M, Las L, Yovel Y, Ulanovsky N (2015) Spatial cognition in bats and rats: from sensory acquisition to multiscale maps and navigation. Nat Rev Neurosci 16:94-108. doi:10.1038/nrn3888

Gilbert PE, Kesner RP (2004) Memory for objects and their locations: the role of the hippocampus in retention of object-place associations.
Neurobiol Learn Mem 81:39-45. doi:10.1016/S1074-7427(03) 00069-8

Golomb JD, Kupitz CN, Thiemann CT (2014a) The influence of object location on identity: a "spatial congruency bias". J Exp Psychol Gen 143:2262-2278. doi:10.1037/xge0000017

Golomb JD, L'Heureux ZE, Kanwisher N (2014b) Feature-binding errors after eye movements and shifts of attention. Psychol Sci 25:10671078. doi:10.1177/0956797614522068

Hampstead BM, Stringer AY, Stilla RF, Amaraneni A, Sathian K (2011) Where did I put that? Patients with amnestic mild cognitive impairment demonstrate widespread reductions in activity during the encoding of ecologically relevant object-location associations. Neuropsychologia 49:2349-2361. doi:10.1016/j.neuropsychologia. 2011.04.008

Hannula DE, Ranganath C (2008) Medial temporal lobe activity predicts successful relational memory binding. J Neurosci 28:116-124. doi: 10.1523/JNEUROSCI.3086-07.2008

Hannula DE, Tranel D, Cohen NJ (2006) The long and the short of it: relational memory impairments in amnesia, even at short lags. J Neurosci 26:8352-8359. doi:10.1523/jneurosci.5222-05.2006

Hartley T, Lever C, Burgess N, O'Keefe J (2014) Space in the brain: how the hippocampal formation supports spatial cognition. Philos Trans R Soc B-Biol Sci 369:18. doi:10.1098/rstb.2012.0510

Jiang Y, Olson IR, Chun MM (2000) Organization of visual short-term memory. J Exp Psychol: Learn Mem Cogn 26:683-702. doi:10. 1037/0278-7393.26.3.683

Jiang Y, Chun M, Olson I (2004) Perceptual grouping in change detection. P\&P 66:446-453. doi:10.3758/BF03194892

Jones EK, Sünram-Lea SI, Wesnes KA (2012) Acute ingestion of different macronutrients differentially enhances aspects of memory and attention in healthy young adults. Biol Psychol 89:477-486. doi:10. 1016/j.biopsycho.2011.12.017

Le Floch J-P, Escuyer P, Baudin E, Baudon D, Perlemuter L (1990) Blood glucose area under the curve: methodological aspects. Diabetes Care 13:172-175. doi:10.2337/diacare.13.2.172

Lee I, Solivan F (2008) The roles of the medial prefrontal cortex and hippocampus in a spatial paired-association task. Learn Mem 15: 357-367. doi:10.1101/lm.902708

Mackay C, Cox T, Burrows G, Lazzerini T (1978) An inventory for measurement of self-reported stress and arousal. Br J Soc Clin Psychol 17:283-284. doi:10.1111/j.2044-8260.1978.tb00280.x

McIntosh RD, Schenk T (2009) Two visual streams for perception and action. Curr Trends Neuropsychologia 47:1391-1396. doi:10.1016/ j.neuropsychologia.2009.02.009

McNay EC, Gold PE (2001) Age-related differences in hippocampal extracellular fluid glucose concentration during behavioral testing and following systemic glucose administration. J Gerontol Series A: Biol Sci Med Sci 56:B66-B71. doi:10.1093/gerona/56.2.B66

McNay EC, Fries TM, Gold PE (2000) Decreases in rat extracellular hippocampal glucose concentration associated with cognitive demand during a spatial task. Proc Natl Acad Sci U S A 97:28812885. doi:10.1073/pnas.050583697

Meikle A, Riby LM, Stollery B (2004) The impact of glucose ingestion and gluco-regulatory control on cognitive performance: a comparison of younger and middle aged adults. Hum Psychopharmacol Clin Exp 19:523-535. doi:10.1002/hup.643

Messier C (2004) Glucose improvement of memory: a review. Eur J Pharmacol 490:33-57. doi:10.1016/j.ejphar.2004.02.043

Mishkin M, Ungerleider LG (1982) Contribution of striate inputs to the visuospatial functions of parieto-preoccipital cortex in monkeys. Behav Brain Res 6:57-77. doi:10.1016/0166-4328(82)90081-X

Mishkin M, Ungerleider LG, Macko KA (1983) Object vision and spatial vision: two cortical pathways. Trends Neurosci 6:414-417. doi:10. 1016/0166-2236(83)90190-X

Mitchell KJ, Johnson MK, Raye CL, D’Esposito M (2000) fMRI evidence of age-related hippocampal dysfunction in feature binding in 
working memory. Cognitive Brain Research 10:197-206. doi:10. 1016/S0926-6410(00)00029-X

Mohanty A, Flint RW Jr (2001) Differential effects of glucose on modulation of emotional and nonemotional spatial memory tasks. Cogn Affect Behav Neurosci 1:90-95. doi:10.3758/CABN.1.1.90

Moscovitch M, Kapur S, Kohler S, Houle S (1995) Distinct neural correlates of visual long-term memory for spatial location and object identity: a positron emission tomography study in humans. Proc Natl Acad Sci U S A 92:3721-3725. doi:10.1073/pnas.92.9.3721

Mumby DG, Gaskin S, Glenn MJ, Schramek TE, Lehmann H (2002) Hippocampal damage and exploratory preferences in rats: memory for objects, places, and contexts. Learn Mem 9:49-57. doi:10.1101/ $\operatorname{lm} .41302$

Nunn JA, Graydon FJX, Polkey CE, Morris RG (1999) Differential spatial memory impairment after right temporal lobectomy demonstrated using temporal titration. Brain 122:47-59. doi:10.1093/brain/ 122.1.47

O'Keefe J, Nadel L (1978) The hippocampus as a cognitive map. Oxford University Press, Oxford

Olsen RK, Moses SN, Riggs L, Ryan JD (2012) The hippocampus supports multiple cognitive processes through relational binding and comparison. Front Hum Neurosci 6:146. doi:10.3389/fnhum.2012. 00146

Olsen RK, Rondina Ii R, Riggs L, Meltzer JA, Ryan JD (2013) Hippocampal and neocortical oscillatory contributions to visuospatial binding and comparison. J Exp Psychol Gen 142:1335-1345. doi:10.1037/a0034043

Olson IR, Page K, Sledge Moore K, Chatterjee A, Verfaellie M (2006) Working memory for conjunctions relies on the medial temporal lobe. J Neurosci 26:4596-4601. doi:10.1523/JNEUROSCI.192305.2006

Owen L, Scholey AB, Finnegan Y, Hu H, Sünram-Lea SI (2012) The effect of glucose dose and fasting interval on cognitive function: a double-blind, placebo-controlled, six-way crossover study. Psychopharmacology 220:577-589. doi:10.1007/s00213-0112510-2

Owen L, Scholey A, Finnegan Y, Sunram-Lea SI (2013) Response variability to glucose facilitation of cognitive enhancement. Br J Nutr 110:1873-1884. doi:10.1017/s0007114513001141

Perfect TJ, Mayes AR, Downes JJ, Van Eijk R (1996) Does context discriminate recollection from familiarity in recognition memory? Q J Exp Psychol 49:797-813. doi:10.1080/027249896392603

Pertzov Y, Husain M (2014) The privileged role of location in visual working memory. Atten Percept Psychophys 76:1914-1924. doi: 10.3758/s13414-013-0541-y

Pertzov Y, Dong MY, Peich M-C, Husain M (2012) Forgetting what was where: the fragility of object-location binding. PLoS One 7, e48214. doi:10.1371/journal.pone.0048214

Pertzov Y, Miller TD, Gorgoraptis N, Caine D, Schott JM, Butler C, Husain M (2013) Binding deficits in memory following medial temporal lobe damage in patients with voltage-gated potassium channel complex antibody-associated limbic encephalitis. Brain 136:2474 2485. doi:10.1093/brain/awt129

Piekema C, Kessels RPC, Mars RB, Petersson KM, Fernández G (2006) The right hippocampus participates in short-term memory maintenance of object-location associations. Neuroimage 33:374-382. doi:10.1016/j.neuroimage.2006.06.035

Poch C, Campo P, Parmentier FBR, Ruiz-Vargas JM, Elsley JV, Castellanos NP, Maestú F, del Pozo F (2010) Explicit processing of verbal and spatial features during letter-location binding modulates oscillatory activity of a fronto-parietal network. Neuropsychologia 48:3846-3854. doi:10.1016/j.neuropsychologia. 2010.09.015

Postma A, De Haan EHF (1996) What was where? Memory for object locations. Q J Exp Psychology Section A 49:178-199. doi:10.1080/ 713755605
Postma A, Kessels RPC, van Asselen M (2008) How the brain remembers and forgets where things are: the neurocognition of objectlocation memory. Neurosci Biobehav Rev 32:1339-1345. doi:10. 1016/j.neubiorev.2008.05.001

Prabhakaran V, Narayanan K, Zhao Z, Gabrieli JD (2000) Integration of diverse information in working memory within the frontal lobe. Nat Neurosci 3:85-90. doi:10.1038/71156

Pruessner JC, Kirschbaum C, Meinlschmid G, Hellhammer DH (2003) Two formulas for computation of the area under the curve represent measures of total hormone concentration versus time-dependent change. Psychoneuroendocrinology 28:916-931. doi:10.1016/ S0306-4530(02)00108-7

Rajsic J, Wilson D (2014) Asymmetrical access to color and location in visual working memory. Atten Percept Psychophys 76:1902-1913. doi:10.3758/s13414-014-0723-2

Rao A, Hu H, Nobre AC (2005) The effects of combined caffeine and glucose drinks on attention in the human brain. Nutr Neurosci 8: 141-153. doi:10.1080/10284150500096994

Riby LM (2004) The impact of age and task domain on cognitive performance: a meta-analytic review of the glucose facilitation effect. Brain Impair 5:145-165. doi:10.1375/brim.5.2.145.58253

Riby L, Riby D (2006) Glucose, ageing and cognition: the hippocampus hypothesis. In: Ballesteros S (ed) Ageing, cognition, and neuroscience. UNED, Varia, Madrid, pp 79-92

Riby LM, Sunram-Lea SI, Graham C, Foster JK, Cooper T, Moodie C, Gunn VP (2008) P3b versus P3a: an event-related potential investigation of the glucose facilitation effect. J Psychopharmacol 22:486492. doi:10.1177/0269881107081561

Roth J, Franconeri S (2012) Asymmetric coding of categorical spatial relations in both language and vision. Front Psychol 3:464. doi:10. 3389/fpsyg.2012.00464

Scholey AB, Kennedy DO (2004) Cognitive and physiological effects of an "energy drink": an evaluation of the whole drink and of glucose, caffeine and herbal flavouring fractions. Psychopharmacology 176: 320-330. doi:10.1007/s00213-004-1935-2

Scholey AB, Sunram-Lea SI, Greer J, Elliott J, Kennedy DO (2009) Glucose administration prior to a divided attention task improves tracking performance but not word recognition: evidence against differential memory enhancement? Psychopharmacology 202:549_ 558. doi:10.1007/s00213-008-1387-1

Serra-Grabulosa JM, Adan A, Falcón C, Bargalló N (2010) Glucose and caffeine effects on sustained attention: an exploratory fMRI study. Hum Psychopharmacol Clin Exp 25:543-552. doi:10.1002/hup. 1150

Shastri L (2002) Episodic memory and cortico-hippocampal interactions. Trends Cogn Sci 6:162-168. doi:10.1016/s1364-6613(02)01868-5

Smith MA, Riby LM, Sünram-Lea SI, van Eekelen JAM, Foster JK (2009) Glucose modulates event-related potential components of recollection and familiarity in healthy adolescents. Psychopharmacology 205:11-20. doi:10.1007/s00213-009-1509-4

Smith MA, Riby LM, van Eekelen JAM, Foster JK (2011) Glucose enhancement of human memory: a comprehensive research review of the glucose memory facilitation effect. Neurosci Biobehav Rev 35: 770-783. doi:10.1016/j.neubiorev.2010.09.008

Spellman T, Rigotti M, Ahmari SE, Fusi S, Gogos JA, Gordon JA (2015) Hippocampal-prefrontal input supports spatial encoding in working memory. Nature. doi:10.1038/nature14445

Squire LR (1992) Memory and the hippocampus: a synthesis from findings with rats, monkeys, and humans. Psychol Rev 99:195-231. doi: 10.1037/0033-295X.99.2.195

Stollery B, Christian L (2013) Glucose and memory: the influence of drink, expectancy, and beliefs. Psychopharmacology 228:685-697. doi:10.1007/s00213-013-3074-0

Stollery B, Christian L (2015) Glucose, relational memory, and the hippocampus. Psychopharmacology 232:2113-2125. doi:10.1007/ s00213-014-3842-5 
Sunram-Lea SI, Dewhurst SA, Foster JK (2008) The effect of glucose administration on the recollection and familiarity components of recognition memory. Biol Psychol 77:69-75. doi:10.1016/j. biopsycho.2007.09.006

Sünram-Lea SI, Owen L, Finnegan Y, Hu H (2011) Dose-response investigation into glucose facilitation of memory performance and mood in healthy young adults. J Psychopharmacol 25:1076-1087. doi: $10.1177 / 0269881110367725$

Tomlinson DR, Gardiner NJ (2008) Glucose neurotoxicity. Nat Rev Neurosci 9:36-45. doi:10.1038/nrn2294

Treisman A (1988) Features and objects: the fourteenth Bartlett memorial lecture. Q J Exp Psychol Sect A 40:201-237. doi:10.1080/ 02724988843000104

Treisman A (1996) The binding problem. Curr Opin Neurobiol 6:171178. doi:10.1016/S0959-4388(96)80070-5

Treisman A, Zhang W (2006) Location and binding in visual working memory. Mem Cognit 34:1704-1719. doi:10.3758/BF03195932

Ungerleider LG, Haxby JV (1994) 'What' and 'where' in the human brain. Curr Opin Neurobiol 4:157-165. doi:10.1016/0959-4388(94) 90066-3

Warburton EC, Brown MW (2010) Findings from animals concerning when interactions between perirhinal cortex, hippocampus and medial prefrontal cortex are necessary for recognition memory. Neuropsychologia 48:2262-2272. doi:10.1016/j.neuropsychologia. 2009.12.022
Watson PD, Voss JL, Warren DE, Tranel D, Cohen NJ (2013) Spatial reconstruction by patients with hippocampal damage is dominated by relational memory errors. Hippocampus 23:570-580. doi:10. 1002/hipo.22115

Winocur G (1995) Glucose-enhanced performance by aged rats on a test of conditional discrimination learning. Psychobiology 23:270-276. doi:10.3758/BF03333073

Winocur G, Gagnon S (1998) Glucose treatment attenuates spatial learning and memory deficits of aged rats on tests of hippocampal function. Neurobiol Aging 19:233-241. doi:10.1016/S0197-4580(98) 00057-8

Yee LTS, Hannula DE, Tranel D, Cohen NJ (2014) Short-term retention of relational memory in amnesia revisited: accurate performance depends on hippocampal integrity. Front Hum Neurosci 8:16. doi: 10.3389/fnhum. 2014.00016

Zimmer HD, Speiser HR, Seidler B (2003) Spatio-temporal workingmemory and short-term object-location tasks use different memory mechanisms. Acta Psychologica 114:41-65. doi:10.1016/S00016918(03)00049-0

Zimmer HD, Mecklinger A, Lindenberger U (2006) Levels of binding: types, mechanisms, and functions of binding in remembering. In: Zimmer HD, Mecklinger A, Lindenberger U (eds) Handbook of binding and memory: perspectives from cognitive neuroscience. Oxford University Press, Oxford, pp 3-22 